\title{
Asymptotic Growth of the Local Ground-State Entropy of the Ideal Fermi Gas in a Constant Magnetic Field
}

\author{
Hajo Leschke ${ }^{1}$, Alexander V. Sobolev ${ }^{2}$, Wolfgang Spitzer ${ }^{3}$ (1) \\ ${ }^{1}$ Institut für Theoretische Physik, Universität Erlangen-Nürnberg, Staudtstraße 7, 91058 Erlangen, Germany. \\ E-mail: hajo.leschke@physik.uni-erlangen.de \\ 2 Department of Mathematics, University College London, Gower Street, London WC1E 6BT, UK. \\ E-mail: a.sobolev@ucl.ac.uk \\ 3 Fakultät für Mathematik und Informatik, FernUniversität in Hagen, Universitätsstraße 1, 58097 Hagen, \\ Germany. E-mail: wolfgang.spitzer@fernuni-hagen.de
}

Received: 25 July 2019 / Accepted: 14 July 2020

Published online: 21 December 2020 - (C) The Author(s) 2020

\begin{abstract}
We consider the ideal Fermi gas of indistinguishable particles without spin but with electric charge, confined to a Euclidean plane $\mathbb{R}^{2}$ perpendicular to an external constant magnetic field of strength $B>0$. We assume this (infinite) quantum gas to be in thermal equilibrium at zero temperature, that is, in its ground state with chemical potential $\mu \geq B$ (in suitable physical units). For this (pure) state we define its local entropy $S(\Lambda)$ associated with a bounded (sub)region $\Lambda \subset \mathbb{R}^{2}$ as the von Neumann entropy of the (mixed) local substate obtained by reducing the infinite-area ground state to this region $\Lambda$ of finite area $|\Lambda|$. In this setting we prove that the leading asymptotic growth of $S(L \Lambda)$, as the dimensionless scaling parameter $L>0$ tends to infinity, has the form $L \sqrt{B}|\partial \Lambda|$ up to a precisely given (positive multiplicative) coefficient which is independent of $\Lambda$ and dependent on $B$ and $\mu$ only through the integer part of $(\mu / B-1) / 2$. Here we have assumed the boundary curve $\partial \Lambda$ of $\Lambda$ to be sufficiently smooth which, in particular, ensures that its arc length $|\partial \Lambda|$ is well-defined. This result is in agreement with a so-called area-law scaling (for two spatial dimensions). It contrasts the zero-field case $B=0$, where an additional logarithmic factor $\ln (L)$ is known to be present. We also have a similar result, with a slightly more explicit coefficient, for the simpler situation where the underlying single-particle Hamiltonian, known as the Landau Hamiltonian, is restricted from its natural Hilbert space $\mathrm{L}^{2}\left(\mathbb{R}^{2}\right)$ to the eigenspace of a single but arbitrary Landau level. Both results extend to the whole one-parameter family of quantum Rényi entropies. As opposed to the case $B=0$, the corresponding asymptotic coefficients depend on the Rényi index in a non-trivial way.
\end{abstract}

\section{Contents}

1. Introduction . . . . . . . . . . . . . . . . . . . . . . 674

2. Setting the Stage and Basic Asymptotic Results for Smooth Functions . . . 675

3. Underlying Asymptotic Results for Polynomials . . . . . . . . . . . . . . 681

4. From Smooth Functions to the Entropy Functions . . . . . . . . . . . . . 690 
4.1 Definitions and results . . . . . . . . . . . . . . . . . . 690

4.2 Estimates for singular values . . . . . . . . . . . . . . . . . . . 692

4.3 Proofs of Lemma 7 and Theorem $8 \ldots \ldots$. . . . . . . . . . . . . . . . . . . . . 696

5. On an Improvement to Sub-leading Terms . . . . . . . . . . . . . . . . . 697

A. Roccaforte's Formula for the Area of Intersections . . . . . . . . . . . . . 697

B. Miscellaneous Identities . . . . . . . . . . . . . . . . . . . . 698

B.1 Proof of the result (3.26) of a change of variables . . . . . . . . . . . 698

B.2 Change of variables in Laguerre polynomials . . . . . . . . . . . 702

B.3 Proof of the identity (3.28) . . . . . . . . . . . . 703

\section{Introduction}

Quantum correlations in many-particle ground states occur in a genuine and simple form for fermions without interactions between them. In this case all correlations are exclusively due to the Pauli-Fermi-Dirac statistics and are not affected by classical correlations. This certainly explains why the authors of many recent publications, devoted to the "trendy topic" of entanglement entropy, have considered ground states of free fermions in discrete or continuous position space. For these pure states its (bipartite spatial) entanglement entropy boils down to its local entropy associated with a bounded region $\Lambda$ in the position space. An informal definition of the local entropy is given in the above abstract. For a formal definition, in the present context, see (4.2) (with $\alpha=1$ ) below. This local ground-state entropy may serve as a useful, but rough, single-number quantification of the correlations of all the particles in the region $\Lambda$ with all those outside.

The local ground-state entropy is a complicated function(al) of $\Lambda$ and difficult to study by analytic methods. Even without interactions, one can in general only hope for estimates and/or asymptotic results when the volume of the bounded region becomes large. As discovered by Gioev and Klich [8], one fascinating aspect of these type of asymptotic results is the connection to the quasi-classical evaluation of traces of (truncated) Wiener-Hopf operators (or Toeplitz matrices in the discrete, one-dimensional, case), that is, to a conjecture of Harold Widom (respectively of Fisher and Hartwig). The "Widom conjecture" was finally proved by one of us in [29] and opened the gate to prove a conjecture by Gioev and Klich [8] about the precise asymptotic growth of the local ground-state entropy of free fermions in multi-dimensional Euclidean space, see [16].

Of course, it is physically relevant and mathematically interesting to determine such a precise asymptotics also for ground states of fermions subject to an external field or even with interactions between them. From a rigorous point of view, the latter seems currently to be out of reach. Concerning external scalar fields there are publications devoted to free fermions in a (random) potential [6,19-22] or in a one-dimensional periodic potential [24]. As an aside, we mention that in the case of free fermions the large-scale behavior of the local entropy is not only known for the ground state, but also for the thermal equilibrium state at any temperature $[15,17]$.

In the present paper, we (return to zero temperature and) consider the ground state of non-relativistic, spinless, and electrically charged fermions in the Euclidean plane $\mathbb{R}^{2}$ without interactions between them, but subject to an external magnetic field which is perpendicular to the plane and of constant strength $B>0$. This ground state became of interest in condensed-matter physics at first in the early 1930 s for simplified explanations of the Landau diamagnetism and the De Haas-Van Alphen effect observed in metals, see $[10,23]$. The interest got revived and enhanced after the discovery of the (integrally) 
quantized Hall effect in certain quasi-two-dimensional semiconductor materials by Klaus von Klitzing in the year 1980, see [10,30]. To our knowledge, analytical contributions to the asymptotic growth of the local entropy of this ground state were made by Klich [13], by Rodríguez and Sierra [26,27], and recently by Charles and Estienne [4]. All these authors consider the case of the lowest Landau level only. In addition, [13,26,27] treat regions of simple geometric shape only. The important work of Rodríguez and Sierra [26] contains non-rigorous arguments, but their formula for the asymptotic coefficient $\mathrm{M}_{0}\left(h_{1}\right)$ (see (4.1) and (4.4) for the definition), confirmed in [4], has been a guide for us to arrive at the more general asymptotic coefficients presented here. After all, the simplicity of the coefficient $\mathbf{M}_{\ell}(f)$ of the sub-leading boundary-curve term in (2.13), see (2.9) and (2.10), is striking, given that it results from the $2 m$-fold integration in (3.20) for arbitrary exponent $m \geq 1$.

By adapting Roccaforte's approach for translation invariant integral kernels [25] to those of the Landau-projection operators we extend results in $[4,26]$ to rather general regions, to an arbitrary single Landau-level eigenspace, and even to the orthogonal sum of the first $n+1$ eigenspaces for arbitrary $n \geq 0$. By the last extension we can allow for an arbitrary value of the chemical potential $\mu \geq B$ (in suitable physical units) and, hence, for an arbitrary areal density of the particle number. Our proof consists of two basic steps. In the first step, we present the precise asymptotics of the trace of smooth functions of localized (or spatially truncated) Landau projections. By a suitable application of the Stone-Weierstraß approximation theorem this asymptotics is shown to follow from a corresponding one for polynomials, based on Lemma 5. Our proof of Lemma 5 is elementary in the sense that it does not make use of the quasi-classical functional calculus for pseudo-differential operators, not even of standard stationaryphase evaluation techniques. However, it involves one change of variables which is cumbersome to utilize, see (3.26). In the second step, we show how to get from smooth functions to the Rényi entropy functions $h_{\alpha}$. This is not obvious, in particular for Rényi index $\alpha \leq 1$, and does not follow from standard approximation schemes. Therefore we prove and employ certain Schatten-von Neumann quasi-norm estimates, similarly to what has been done in [16].

Our main result is Theorem 8. It turns out that all local Rényi ground-state entropies grow to leading order proportional to $L$ when the dimensionless parameter $L>0$ of the scaled region $L \Lambda$ is sent to infinity (due to the off-diagonal Gaussian decay of the Landauprojection integral kernels). This is in agreement with the so-called area-law scaling [5]. Given that, the corresponding proportionality factor has the form $\sqrt{B}|\partial \Lambda| \mathbf{M}_{\leq \nu}\left(h_{\alpha}\right)$. Here, the first two factors are expected from considering physical dimensions, because $|\partial \Lambda|$ denotes the arc length of the (smooth) boundary curve $\partial \Lambda$. The third factor $\mathrm{M}_{\leq v}\left(h_{\alpha}\right)$ is a dimensionless asymptotic coefficient precisely given by (2.12) and (4.1). It depends in a non-trivial way on the Rényi index $\alpha>0$, but on $B$ and $\mu$ only through the integer part $v$ of $(\mu / B-1) / 2$. It is finite and positive, but in general a rather complicated expression. However, if $\mu<3 B$, then it simplifies considerably, because only the lowest Landau-level eigenspace remains to be relevant. The result agrees (for $\alpha=1$ ) with the one proved recently by Charles and Estienne [4].

\section{Setting the Stage and Basic Asymptotic Results for Smooth Functions}

We denote the scalar products in the Euclidean plane $\mathbb{R}^{2}$ and in the Hilbert space $L^{2}(\mathrm{D})$ of complex-valued, square-integrable functions on a Borel set $D \subseteq \mathbb{R}^{2}$ by the same 
bracket $\langle\cdot \mid \cdot\rangle$ and use the same notation $\|\cdot\|$ for the induced norms. Our convention is that the scalar product is anti-linear in the first and linear in the second argument.

Since in the ideal Fermi gas the indistinguishable (point) particles do not interact with each other, it is sufficient to consider the common Schrödinger operator for the kinetic energy of a single particle in the plane subject to a perpendicular constant magnetic field of strength $B>0$. This operator is known as the Landau Hamiltonian. It acts selfadjointly on a dense domain of definition in the single-particle Hilbert space $L^{2}\left(\mathbb{R}^{2}\right)$ and is given by

$$
\mathrm{H}:=(-\mathrm{i} \nabla-a)^{2} .
$$

Here, we choose the symmetric gauge $a(x)=\left(a_{1}(x), a_{2}(x)\right):=\left(x_{2},-x_{1}\right) B / 2$ for the vector potential $a: \mathbb{R}^{2} \rightarrow \mathbb{R}^{2}$ generating the constant magnetic field (vector) perpendicular to the plane with Cartesian coordinates $x=\left(x_{1}, x_{2}\right)$. Other gauges yield operators being unitarily equivalent to $\mathrm{H}$. Moreover, here and in the following we are using physical units such that the particle mass and the particle charge equals $1 / 2$ and 1 , respectively. Similarly, we put the speed of light, Planck's constant (divided by $2 \pi$ ), and Boltzmann's constant all equal to 1 .

Throughout the paper we use the symplectic $2 \times 2$ matrix $\mathrm{J}:=\left[\begin{array}{cc}0 & 1 \\ -1 & 0\end{array}\right]$, the generalized Laguerre polynomials

$$
\mathcal{L}_{\ell}^{(k)}(t):=\sum_{j=0}^{\ell} \frac{(-1)^{j}}{j !}\left(\begin{array}{c}
\ell+k \\
\ell-j
\end{array}\right) t^{j}, \quad k \in\{-\ell,-\ell+1, \ldots\}, \quad t \geq 0
$$

of degree $\ell \in \mathbb{N}_{0}$, and the abbreviation $\mathcal{L}_{\ell}:=\mathcal{L}_{\ell}^{(0)}$. For each degree $\ell$ we define an infinite-dimensional projection (operator) $\mathrm{P}_{\ell}$ on $\mathrm{L}^{2}\left(\mathbb{R}^{2}\right)$ by the Hermitian integral kernel

$p_{\ell}(x, y):=\frac{B}{2 \pi} \exp \left(-B\|x-y\|^{2} / 4\right) \mathcal{L}_{\ell}\left(B\|x-y\|^{2} / 2\right) \exp \left(\mathrm{i} \frac{B}{2}\langle x \mid \mathrm{J} y\rangle\right), \quad x, y \in \mathbb{R}^{2}$.

It is obviously $\mathrm{C}^{\infty}$-smooth and a Carleman kernel in the sense that it is square integrable with respect to $y \in \mathbb{R}^{2}$ for all $x \in \mathbb{R}^{2}$, and vice versa. Now the spectral decomposition of the Landau Hamiltonian $\mathrm{H}$ may be written as

$$
\mathrm{H}=B \sum_{\ell=0}^{\infty}(2 \ell+1) \mathrm{P}_{\ell}
$$

As usual, this formula is meant in the sense of strong operator convergence on $\mathrm{L}^{2}\left(\mathbb{R}^{2}\right)$. It goes back to Fock [7] and Landau [14]. The projections $\mathrm{P}_{\ell}$, now recognizable as spectral projections, depend on the chosen gauge through the last (complex-valued phase) factor in (2.2), but the set $\{B, 3 B, 5 B, \ldots\}$ of harmonic-oscillator like eigenvalues, in other words Landau levels, does not. The degree $\ell$ is now called Landau-level index. We will also need the projection $\mathrm{P}_{\leq n}:=\sum_{0 \leq \ell \leq n} \mathrm{P}_{\ell}$ on the orthogonal sum of the first $n+1$ Landau-level eigenspaces $\mathrm{P}_{\ell} \mathrm{L}^{2}\left(\mathbb{R}^{2}\right)$ and mention the functional relation $\mathcal{L}_{\leq n}:=$ $\sum_{0 \leq \ell \leq n} \mathcal{L}_{\ell}=\mathcal{L}_{n}^{(1)}$. For later purposes we single out the (translation invariant) Gaussian part of the kernel by defining 


$$
\begin{aligned}
g(z) & :=\left(\frac{B}{2 \pi}\right)^{1 / 2} \exp \left(-\frac{B z^{2}}{4}\right), \quad z \in \mathbb{R}, \\
g_{2}(x) & :=g\left(x^{\prime}\right) g\left(x^{\prime \prime}\right), \quad x=\left(x^{\prime}, x^{\prime \prime}\right) \in \mathbb{R}^{2},
\end{aligned}
$$

so that

$$
\begin{aligned}
\mathrm{P}_{\ell}(x, y) & \equiv p_{\ell}(x, y)=\mathcal{L}_{\ell}\left(B\|x-y\|^{2} / 2\right) g_{2}(x-y) \exp \left(\mathrm{i} \frac{B}{2}\langle x \mid \mathrm{J} y\rangle\right), \\
\mathrm{P}_{\leq n}(x, y) & :=\sum_{\ell=0}^{n} p_{\ell}(x, y)=\mathcal{L}_{\leq n}\left(B\|x-y\|^{2} / 2\right) g_{2}(x-y) \exp \left(\mathrm{i} \frac{B}{2}\langle x \mid \mathrm{J} y\rangle\right) .
\end{aligned}
$$

Now we are prepared to turn to the ground state of the ideal Fermi gas with the Landau Hamiltonian, see (2.1) and (2.3), as its single-particle Hamiltonian and with the chemical potential $\mu \geq B$ as a real parameter. According to the grand-canonical formalism of quantum statistical mechanics $[3,10]$ this (infinite-area) ground state is quasi-Gaussian (in other words, quasi-free) and, as such, characterized by its reduced single-particle density operator on $\mathrm{L}^{2}\left(\mathbb{R}^{2}\right)$ given by the Fermi projection

$$
\Theta(\mu \mathbb{1}-\mathrm{H})=\sum_{\ell=0}^{\infty} \Theta(\mu-(2 \ell+1) B) \mathrm{P}_{\ell}=\mathrm{P}_{\leq \nu}, \quad \mu \geq B .
$$

Here, $\Theta$ is Heaviside's unit-step function (defined by $\Theta(t):=1$ if $t \geq 0$ and zero otherwise), $\mathbb{1}$ denotes the identity operator on $\mathrm{L}^{2}\left(\mathbb{R}^{2}\right)$, and $v:=\lfloor(\mu / B-1) / 2\rfloor$ is the integer part of $(\mu / B-1) / 2 \geq 0$. Now we consider a Borel set $\Lambda \subseteq \mathbb{R}^{2}$ and the multiplication operator $\mathbb{1}_{\Lambda}$ on $\mathrm{L}^{2}\left(\mathbb{R}^{2}\right)$ corresponding to its indicator function $1_{\Lambda}$ on $\mathbb{R}^{2}$. Moreover, we introduce the local(ized) Landau projections

$$
\mathrm{P}_{\ell}(\Lambda):=\mathbb{1}_{\Lambda} \mathrm{P}_{\ell} \mathbb{1}_{\Lambda}, \mathrm{P}_{\leq n}(\Lambda):=\mathbb{1}_{\Lambda} \mathrm{P}_{\leq n} \mathbb{1}_{\Lambda}
$$

The quasi-Gaussian substate associated with $\Lambda \subseteq \mathbb{R}^{2}$ is now simply characterized by the local(ized) Fermi projection

$$
\mathbb{1}_{\Lambda} \Theta(\mu \mathbb{1}-\mathrm{H}) \mathbb{1}_{\Lambda}=\mathrm{P}_{\leq v}(\Lambda),
$$

see [9].

We ignore the uninteresting case $\mu<B$, because then the Fermi projection is the zero operator corresponding to a vanishing number of particles. In contrast, as a function of $\mu \geq B$ the (mean) local areal density of the particle number, $\rho(x)$, in the ground state characterized by the density operator $(2.5)$ is non-zero and equal to the diagonal of its integral kernel, that is,

$$
\rho(x)=\mathrm{P}_{\leq \nu}(\Lambda)(x, x)=(\nu+1) \frac{B}{2 \pi} 1_{\Lambda}(x), \quad x \in \mathbb{R}^{2} .
$$

Integration over the plane $\mathbb{R}^{2}$ gives the (mean) total number of particles, $(v+1) B|\Lambda| / 2 \pi$, in its subset $\Lambda$ with (Lebesgue) area $|\Lambda|$. So $v+1$ corresponds to an integer value of the filling factor in the physics literature. In view of (2.5) it suffices in the following to consider the projection $\mathrm{P}_{\leq n}(\Lambda)$ for arbitrary $n \in \mathbb{N}_{0}$. Moreover, from now on we typically assume that $\Lambda \subset \mathbb{R}^{2}$ is the union of finitely many bounded domains (open connected sets), such that their closures are pairwise disjoint. We call such a $\Lambda$ a bounded region. 
If the boundary curve $\partial \Lambda$ of $\Lambda$ is $C^{\gamma}, \gamma \in \mathbb{N} \cup\{\infty\}$, then we say that $\Lambda$ is a bounded $\mathrm{C}^{\gamma}$-region.

Before we state our basic asymptotic results we recall the definition of the Hermite polynomials, $H_{\ell}$, of degree $\ell \in \mathbb{N}_{0}$. They satisfy the orthogonality relation

$$
\int_{\mathbb{R}} \mathrm{d} t \exp \left(-t^{2}\right) H_{\ell}(t) H_{\ell^{\prime}}(t)=\sqrt{\pi} 2^{\ell} \ell ! \delta_{\ell, \ell^{\prime}}, \quad \ell, \ell^{\prime} \in \mathbb{N}_{0} .
$$

An explicit formula is

$$
H_{\ell}(t)=\ell ! \sum_{j=0}^{\lfloor\ell / 2\rfloor} \frac{(-1)^{j}}{j !(\ell-2 j) !}(2 t)^{\ell-2 j}, \quad t \in \mathbb{R}, \ell \in \mathbb{N}_{0}
$$

The Hermite functions $\psi_{\ell}$, defined by

$$
\psi_{\ell}(t):=\left(\sqrt{\pi} 2^{\ell} \ell !\right)^{-1 / 2} H_{\ell}(t) \exp \left(-t^{2} / 2\right), \quad t \in \mathbb{R}, \ell \in \mathbb{N}_{0},
$$

constitute an orthonormal basis of the Hilbert space $\mathrm{L}^{2}(\mathbb{R})$ and are the (energy) eigenfunctions of the one-dimensional harmonic oscillator, that is,

$$
-\psi_{\ell}^{\prime \prime}(t)+t^{2} \psi_{\ell}(t)=(2 \ell+1) \psi_{\ell}(t), \quad t \in \mathbb{R} .
$$

For $\xi \in \mathbb{R}$ and a complex-valued function $f$ on the closed unit interval $[0,1]$ as in Lemma 3 below we define

$$
\lambda_{\ell}(\xi):=\int_{\xi}^{\infty} \mathrm{d} t \psi_{\ell}(t)^{2}, \quad \mathrm{M}_{\ell}(f):=\int_{\mathbb{R}} \frac{\mathrm{d} \xi}{2 \pi}\left[f\left(\lambda_{\ell}(\xi)\right)-f(1) \lambda_{\ell}(\xi)\right], \quad \ell \in \mathbb{N}_{0} .
$$

Obviously, each function $\lambda_{\ell}$ takes values in $[0,1]$ and is (strictly) decreasing. We also need to introduce for each $n \in \mathbb{N}_{0}$ the one-parameter family of operators

$$
\mathcal{K}_{n, \xi}:=\sum_{\ell=0}^{n}\left|\psi_{\ell, \xi}\right\rangle\left\langle\psi_{\ell, \xi}\left|=\mathbb{1}_{[\xi, \infty)} \sum_{\ell=0}^{n}\right| \psi_{\ell}\right\rangle\left\langle\psi_{\ell}\right| \mathbb{1}_{[\xi, \infty)}, \quad \xi \in \mathbb{R}
$$

The operator $\mathcal{K}_{n, \xi}$ maps $\mathrm{L}^{2}(\mathbb{R})$ self-adjointly on its $(n+1)$-dimensional subspace spanned by the first $(n+1)$ truncated Hermite functions $\psi_{\ell, \xi}:=\psi_{\ell} 1_{[\xi, \infty)}$. This operator is not a projection, but it satisfies $0 \leq \mathcal{K}_{n, \xi} \leq \mathbb{1}_{[\xi, \infty)} \leq \mathbb{1}$. Its integral kernel is given by the $\operatorname{sum} \sum_{0 \leq \ell \leq n} \psi_{\ell, \xi}(t) \psi_{\ell, \xi}\left(t^{\prime}\right)$, which can be evaluated explicitly, see (3.36) below.

Along with $\mathrm{M}_{\ell}(f)$ we also define for $n \in \mathbb{N}_{0}$

$$
\mathrm{M}_{\leq n}(f):=\int_{\mathbb{R}} \frac{\mathrm{d} \xi}{2 \pi}\left[\operatorname{tr} f\left(\mathcal{K}_{n, \xi}\right)-f(1) \operatorname{tr} \mathcal{K}_{n, \xi}\right], \quad \mathbf{M}_{\leq 0}(f)=\mathrm{M}_{0}(f) .
$$

Here the trace refers to operators on $\mathrm{L}^{2}(\mathbb{R})$. Now we are in a position to present our two basic asymptotic results. 
Theorem 1 (For the $\ell$ th Landau level, $\ell \in \mathbb{N}_{0}$ ). Let $\Lambda \subset \mathbb{R}^{2}$ be a bounded $\mathrm{C}^{3}$-region in the sense defined below (2.6). Moreover, let $f:[0,1] \rightarrow \mathbb{C}$ be a complex-valued continuous function on the closed unit interval with $f(0)=0$, differentiable from the right at $t=0$ and differentiable from the left at $t=1$. Finally, let $L>0$ be $a$ (dimensionless) scaling parameter. Then we have

$$
\operatorname{tr} f\left(\mathrm{P}_{\ell}(L \Lambda)\right)=L^{2} B \frac{|\Lambda|}{2 \pi} f(1)+L \sqrt{B}|\partial \Lambda| \mathrm{M}_{\ell}(f)+o(L),
$$

as $L \rightarrow \infty$. The asymptotic coefficient is finite, that is, $\left|\mathrm{M}_{\ell}(f)\right|<\infty$.

Here the trace refers to operators on $\mathrm{L}^{2}\left(\mathbb{R}^{2}\right)$ and, as usual, $o(L)$ stands for some function of $L$ with $\lim \sup _{L \rightarrow \infty}|o(L)| / L=0$.

Theorem 2 (For the first $(n+1)$ Landau levels, $n \in \mathbb{N}_{0}$ ). Under the same assumptions as in Theorem 1 we have

$$
\operatorname{tr} f\left(\mathrm{P}_{\leq n}(L \Lambda)\right)=L^{2} B \frac{|\Lambda|}{2 \pi}(n+1) f(1)+L \sqrt{B}|\partial \Lambda| \mathrm{M}_{\leq n}(f)+o(L),
$$

as $L \rightarrow \infty$. The asymptotic coefficient is finite, that is, $\left|\mathrm{M}_{\leq n}(f)\right|<\infty$.

The finiteness of the coefficients $\mathbf{M}_{\ell}(f)$ and $\mathbf{M}_{\leq n}(f)$ are consequences of the following Lemmas 3 and 4, because the smooth function $f$ assumed in Theorems 1 and 2 satisfies the bound (2.15). The proofs of (2.13) and (2.14) are postponed until the proof of Lemma 4. In the next lemma and in the following, by $C, c$ with or without indices, we denote various finite and positive constants, whose precise values are of no importance.

Lemma 3. Let $f:[0,1] \rightarrow \mathbb{C}$ be a measurable function satisfying the bound

$$
|f(t)-f(1) t| \leq C t^{q}(1-t)^{q}, \quad t \in[0,1],
$$

with some $q>0$. Then $\left|\mathrm{M}_{\ell}(f)\right|<\infty$ for all $\ell \in \mathbb{N}_{0}$.

Proof. Firstly, we observe that

$$
\left|\psi_{\ell}(t)\right| \leq C(1+|t|)^{\ell} \mathrm{e}^{-\frac{t^{2}}{2}}, \quad t \in \mathbb{R}, \ell \in \mathbb{N}_{0},
$$

with a constant $C$ depending on $\ell$. Therefore, for $\xi \geq 0$ the function $\lambda_{\ell}$ satisfies the bound

$$
\lambda_{\ell}(\xi) \leq C \int_{\xi}^{\infty} \mathrm{d} t(1+t)^{2 \ell} \mathrm{e}^{-t^{2}} \leq C_{\delta} \mathrm{e}^{-\delta \xi^{2}},
$$

with an arbitrary $\delta<1$. Similarly, for $\xi<0$ we have

$$
\lambda_{\ell}(\xi)=1-\lambda_{\ell}(-\xi) \geq 1-C_{\delta} \mathrm{e}^{-\delta \xi^{2}} .
$$

Combining this with (2.15) yields the claimed result.

Concerning the other coefficient $\mathbf{M}_{\leq n}(f)$ we have the following

Lemma 4. Under the same assumption as in Lemma 3 we have

$$
\left\|f\left(\mathcal{K}_{n, \xi}\right)-f(1) \mathcal{K}_{n, \xi}\right\|_{1} \leq C_{\delta} \mathrm{e}^{-\delta q \xi^{2}},
$$

for every $n \in \mathbb{N}_{0}$ with an arbitrary $0<\delta<1$, and hence $\left|\mathrm{M}_{\leq n}(f)\right|<\infty$. 
Before proving this lemma we compile, for the reader's convenience, some basic properties of the Schatten-von Neumann classes, $\mathfrak{S}_{p}, 0<p<\infty$, of compact operators, see $[2,28]$. By $s_{n}(\mathrm{~T})$ with $n \in \mathbb{N}$, we denote the singular values of a compact operator $\mathrm{T}$ on an abstract (separable) Hilbert space, enumerated in decreasing order. Then, the operator $\mathrm{T}$ is said to belong to $\mathfrak{S}_{p}$ if it has the finite Schatten-von Neumann (quasi-)norm

$$
\|\mathrm{T}\|_{p}:=\left[\sum_{n=1}^{\infty} s_{n}(\mathrm{~T})^{p}\right]^{\frac{1}{p}}<\infty .
$$

If $p \geq 1$, then $\|\cdot\|_{p}$ is a norm. If $0<p<1$, then it is a quasi-norm which satisfies the p-triangle inequality

$$
\left\|\mathrm{T}_{1}+\mathrm{T}_{2}\right\|_{p}^{p} \leq\left\|\mathrm{T}_{1}\right\|_{p}^{p}+\left\|\mathrm{T}_{2}\right\|_{p}^{p} .
$$

The class $\mathfrak{S}_{1}$ is the standard trace class. For $\mathrm{T} \in \mathfrak{S}_{1}$ its trace, $\operatorname{tr} \mathrm{T}$, is well-defined and satisfies $|\operatorname{tr} \mathrm{T}| \leq\|\mathrm{T}\|_{1}$. If $\mathrm{T} \geq 0$, then $\operatorname{tr} \mathrm{T}=\|\mathrm{T}\|_{1}$. We also note that the usual (uniform) operator norm $\|\cdot\|$ may be viewed as $\|\cdot\|_{p}$ in the limit $p \rightarrow \infty$. Finally, we mention that $\|\cdot\|_{p}$ satisfies a Hölder-type inequality in the sense that

$$
\left\|\mathrm{T}_{1} \mathrm{~T}_{2}\right\|_{p} \leq\left\|\mathrm{T}_{1}\right\|_{p_{1}}\left\|\mathrm{~T}_{2}\right\|_{p_{2}}
$$

for any $p_{1}, p_{2} \in(0, \infty]$ with $1 / p_{1}+1 / p_{2}=1 / p$.

Proof. (Proof of Lemma 4) Let us now consider the operator $\mathcal{K}_{n, \xi}=\sum_{\ell=0}^{n} Q_{\ell, \xi}$ defined in (2.11), where we have put $Q_{\ell, \xi}:=\left|\psi_{\ell, \xi}\right\rangle\left\langle\psi_{\ell, \xi}\right|$ With $\tilde{g}(t):=t(1-t)$ we then have

$$
\tilde{g}\left(\mathcal{K}_{n, \xi}\right)=\sum_{\ell=0}^{n}\left(Q_{\ell, \xi}-Q_{\ell, \xi}^{2}\right)-\sum_{\ell, \ell^{\prime}=0, \ell \neq \ell^{\prime}}^{n} Q_{\ell, \xi} Q_{\ell^{\prime}, \xi} .
$$

Since each operator $Q_{\ell, \xi}$ is one-dimensional, we easily find that

$$
\left\|Q_{\ell, \xi}-Q_{\ell, \xi}^{2}\right\|=\lambda_{\ell}(\xi)\left(1-\lambda_{\ell}(\xi)\right) \leq C_{\delta} \mathrm{e}^{-\delta \xi^{2}}, \xi \in \mathbb{R},
$$

according to the proof of Lemma 3. Furthermore,

$$
\left\|Q_{\ell, \xi} Q_{\ell^{\prime}, \xi}\right\|=\left|\left\langle\psi_{\ell, \xi} \mid \psi_{\ell^{\prime}, \xi}\right\rangle\right|\left\|\psi_{\ell, \xi}\right\|\left\|\psi_{\ell^{\prime}, \xi}\right\| \leq\left|\left\langle\psi_{\ell, \xi} \mid \psi_{\ell^{\prime}, \xi}\right\rangle\right| .
$$

Consequently, for $\xi \geq 0$, we have

$$
\left\|Q_{\ell, \xi} Q_{\ell^{\prime}, \xi}\right\| \leq\left\|\psi_{\ell, \xi}\right\|\left\|\psi_{\ell^{\prime}, \xi}\right\| \leq \sqrt{\lambda_{\ell}(\xi) \lambda_{\ell^{\prime}}(\xi)} \leq C_{\delta} \mathrm{e}^{-\delta \xi^{2}} .
$$

For the case $\xi<0$ we observe that $\left\langle\psi_{\ell} \mid \psi_{\ell^{\prime}}\right\rangle=0, \ell \neq \ell^{\prime}$, so that $\left\langle\psi_{\ell, \xi} \mid \psi_{\ell^{\prime}, \xi}\right\rangle=$ $-\left\langle\psi_{\ell}-\psi_{\ell, \xi} \mid \psi_{\ell^{\prime}}-\psi_{\ell^{\prime}, \xi}\right\rangle$, and hence

$$
\left\|Q_{\ell, \xi} Q_{\ell^{\prime}, \xi}\right\| \leq \sqrt{\lambda_{\ell}(-\xi) \lambda_{\ell^{\prime}}(-\xi)} \leq C_{\delta} \mathrm{e}^{-\delta \xi^{2}} .
$$

Collecting the above bounds we conclude that

$$
\left\|\tilde{g}\left(\mathcal{K}_{n, \xi}\right)\right\| \leq C_{\delta} \mathrm{e}^{-\delta \xi^{2}} .
$$

Using now (2.15) we have

$$
\left\|f\left(\mathcal{K}_{n, \xi}\right)-f(1) \mathcal{K}_{n, \xi}\right\|_{1} \leq C\left\|\tilde{g}\left(\mathcal{K}_{n, \xi}\right)\right\|_{q}^{q} .
$$

Since the operator $\mathcal{K}_{n, \xi}$ has finite dimension $n+1$, the right-hand side of the last inequality is bounded from above by $C\left\|\tilde{g}\left(\mathcal{K}_{n, \xi}\right)\right\|^{q}$. Therefore (2.18) leads to the claimed result. 
Proofs of Theorem 1 and Theorem 2. By linearity, Lemmas 5 and 6 in the next section imply Theorems 1 and 2, respectively, for an arbitrary polynomial $f$ (with $f(0)=0$ ). So here we only need to show how to extend the claimed results (2.13) and (2.14) from polynomials to the smooth function $f$ assumed in Theorems 1 and 2. This is by now standard and several versions of this extension are available, e.g. [24,25,29,31]. Here we follow the recent one in [24]. As a by-product we get the a-priori finiteness of the left-hand side of (2.13), see (2.19) and (2.20). The a-priori finiteness of the left-hand side of (2.14) follows similarly.

Without loss of generality we may assume that $f$ is real-valued. Besides the necessary condition $f(0)=0$ we may assume that $f(1)=0$. This can be achieved by replacing $f(t)$ with $f(t)-f(1) t$. The function $f$ has the form $f=b \tilde{g}$ with $\tilde{g}(t)=t(1-t)$, from above, and with some real-valued continuous function $b$ on $[0,1]$. According to the Stone-Weierstraß approximation theorem, there exists for any given $\varepsilon>0$ a real-valued polynomial $p$ on $[0,1]$ such that $\sup _{t \in[0,1]}|p(t)-b(t)| \leq \varepsilon$. Thus with $\tilde{p}:=\tilde{g} p$ we have

$$
\tilde{p}(t)-\varepsilon \tilde{g}(t) \leq f(t) \leq \tilde{p}(t)+\varepsilon \tilde{g}(t), \quad t \in[0,1],
$$

and hence

$$
\operatorname{tr} \tilde{p}\left(\mathrm{P}_{\ell}(L \Lambda)\right)-\varepsilon \operatorname{tr} \tilde{g}\left(\mathrm{P}_{\ell}(L \Lambda)\right) \leq \operatorname{tr} f\left(\mathrm{P}_{\ell}(L \Lambda)\right) \leq \operatorname{tr} \tilde{p}\left(\mathrm{P}_{\ell}(L \Lambda)\right)+\varepsilon \operatorname{tr} \tilde{g}\left(\mathrm{P}_{\ell}(L \Lambda)\right) .
$$

Using (2.13) for $\tilde{p}$ and $\tilde{g}$, we arrive at the bound

$$
\limsup _{L \rightarrow \infty} \frac{\operatorname{tr} f\left(\mathrm{P}_{\ell}(L \Lambda)\right)}{L \sqrt{B}|\partial \Lambda|} \leq \mathrm{M}_{\ell}(\tilde{p})+\varepsilon \mathrm{M}_{\ell}(\tilde{g}) .
$$

Since $\mathrm{M}_{\ell}(\tilde{p})=\mathrm{M}_{\ell}(f)+\mathrm{M}_{\ell}(\tilde{g}(p-b)) \leq \mathrm{M}_{\ell}(f)+\varepsilon \mathrm{M}_{\ell}(\tilde{g})$, the right-hand side of the above inequality does not exceed $\mathrm{M}_{\ell}(f)+2 \varepsilon \mathrm{M}_{\ell}(\tilde{g})$. Similarly,

$$
\liminf _{L \rightarrow \infty} \frac{\operatorname{tr} f\left(\mathrm{P}_{\ell}(L \Lambda)\right)}{L \sqrt{B}|\partial \Lambda|} \geq \mathrm{M}_{\ell}(f)-2 \varepsilon \mathrm{M}_{\ell}(\tilde{g}) .
$$

Since $\varepsilon$ is arbitrary, formula (2.13) follows.

In order to prove Theorem 2 we use the same argument as above for the operator $\mathrm{P}_{\leq n}(L \Lambda)$ and the asymptotic coefficient $\mathrm{M}_{\leq n}(f)$.

\section{Underlying Asymptotic Results for Polynomials}

We have seen that the asymptotic results of Theorems 1 and 2 rely on corresponding results for polynomials $f$. By linearity, it suffices to consider natural powers of the corresponding projections. We begin with

Lemma 5. Let $\Lambda \subset \mathbb{R}^{2}$ be a bounded $\mathrm{C}^{3}$-region and $m \in \mathbb{N}$. Then we have for any $\ell \in \mathbb{N}_{0}$

$$
\operatorname{tr} \mathrm{P}_{\ell}(L \Lambda)^{m}=L^{2} B \frac{|\Lambda|}{2 \pi}+L \sqrt{B}|\partial \Lambda| \int_{\mathbb{R}} \frac{\mathrm{d} \xi}{2 \pi}\left[\lambda_{\ell}(\xi)^{m}-\lambda_{\ell}(\xi)\right]+\mathcal{O}(1),
$$

as $L \rightarrow \infty$. Here, as usual, $\mathcal{O}(1) \equiv \mathcal{O}\left(L^{0}\right)$ stands for some function of $L$ with lim $\sup _{L \rightarrow \infty}|\mathcal{O}(1)|<\infty$. 
Proof. At first we note that $\mathbb{1}_{\Lambda} \mathrm{P}_{\ell}$ is a Hilbert-Schmidt operator on $\mathrm{L}^{2}\left(\mathbb{R}^{2}\right)$, equivalently $\left\|\mathbb{1}_{\Lambda} \mathrm{P}_{\ell}\right\|_{2}<\infty$, because its integral kernel $1_{\Lambda}(x) p_{\ell}(x, y)$ is square-integrable. Therefore $\mathrm{P}_{\ell}(\Lambda)=\left(\mathbb{1}_{\Lambda} \mathrm{P}_{\ell}\right)\left(\mathrm{P}_{\ell} \mathbb{1}_{\Lambda}\right)$ is a trace-class operator and so are its natural powers, due to $\left\|\mathrm{P}_{\ell}(\Lambda)\right\| \leq 1$. The trace of the power $\mathrm{P}_{\ell}(\Lambda)^{m}$ can be calculated by integrating the diagonal of its integral kernel, that is,

$$
\operatorname{tr} \mathrm{P}_{\ell}(\Lambda)^{m}=\int_{\mathbb{R}^{2}} \mathrm{~d} x \mathrm{P}_{\ell}(\Lambda)^{m}(x, x)
$$

This follows from the continuity of $\mathrm{P}_{\ell}(\Lambda)^{m}(x, y)$ as a function of $(x, y) \in \Lambda \times \Lambda$ which, in turn, follows from the m-fold iteration of $\mathrm{P}_{\ell}(\Lambda)(x, y)=p_{\ell}(x, y) 1_{\Lambda}(x) 1_{\Lambda}(y)$ and the dominated-convergence theorem. If $x \notin \Lambda$ or $y \notin \Lambda$, then $\mathrm{P}_{\ell}(\Lambda)^{m}(x, y)=0$. We proceed with (3.2). Since (3.1) is now seen to be true for $m=1$, we assume from now on $m \geq 2$. Then the diagonal $\mathrm{P}_{\ell}(\Lambda)^{m}(x, x)$ is given by

$$
\begin{aligned}
& 1_{\Lambda}(x) \int_{\mathbb{R}^{2(m-1)}} \mathrm{d} x_{1} \cdots \mathrm{d} x_{m-1} p_{\ell}\left(x, x_{1}\right) p_{\ell}\left(x_{1}, x_{2}\right) \cdots p_{\ell}\left(x_{m-2},\right. \\
& \left.x_{m-1}\right) p_{\ell}\left(x_{m-1}, x\right) 1_{\Lambda}\left(x_{1}\right) \cdots 1_{\Lambda}\left(x_{m-1}\right) .
\end{aligned}
$$

It is convenient to change to new integration variables $\mathbf{y}:=\left(y_{1}, \ldots, y_{m-1}\right)$ defined by $y_{1}:=x-x_{1}, y_{2}:=x_{1}-x_{2}, \ldots, y_{m-1}:=x_{m-2}-x_{m-1}$. Furthermore, we set $y_{m}:=y_{1}+\cdots+y_{m-1}$. Then, $x_{1}=x-y_{1}, x_{2}=x-y_{1}-y_{2}, \ldots, x_{m-1}=x-y_{m}$ and

$$
\begin{aligned}
\left\langle x \mid \mathrm{J} x_{1}\right\rangle & =-\left\langle x \mid \mathrm{J} y_{1}\right\rangle \\
\left\langle x_{1} \mid \mathrm{J} x_{2}\right\rangle & =-\left\langle x-y_{1} \mid \mathrm{J} y_{2}\right\rangle \\
\left\langle x_{2} \mid \mathrm{J} x_{3}\right\rangle & =-\left\langle x-y_{1}-y_{2} \mid \mathrm{J} y_{3}\right\rangle \\
\vdots & \vdots \\
\left\langle x_{m-2} \mid \mathrm{J} x_{m-1}\right\rangle & =-\left\langle x-y_{1}-\cdots-y_{m-2} \mid \mathrm{J} y_{m-1}\right\rangle \\
\left\langle x_{m-1} \mid \mathrm{J} x\right\rangle & =-\left\langle y_{m} \mid \mathrm{J} x\right\rangle .
\end{aligned}
$$

With $x_{0}:=x_{m}:=x$ we therefore have

$$
\sum_{i=0}^{m-1}\left\langle x_{i} \mid \mathrm{J} x_{i+1}\right\rangle=\sum_{i=1}^{m-2}\left\langle\sum_{j=1}^{i} y_{j} \mid \mathrm{J} y_{i+1}\right\rangle
$$

If $m=2$ then the left-hand side of (3.4) is zero and its right-hand side is meant to be 0 . By combining (3.2), (3.3), (2.2), and (3.4) the trace of $\mathrm{P}_{\ell}(\Lambda)^{m}$ can now be written as

$$
\operatorname{tr} \mathrm{P}_{\ell}(\Lambda)^{m}=\int_{\mathbb{R}^{2(m-1)}} \mathrm{d} \mathbf{y} \mathfrak{f}_{m}(\mathbf{y}) \int_{\mathbb{R}^{2}} \mathrm{~d} x 1_{\Lambda}(x) 1_{\Lambda}\left(x-y_{1}\right) \cdots 1_{\Lambda}\left(x-y_{m}\right),
$$

with the function $\mathfrak{f}_{m}$ defined by

$$
\mathfrak{f}_{m}(\mathbf{y}):=\left[\prod_{j=1}^{m} \mathcal{L}_{\ell}\left(B\left\|y_{j}\right\|^{2} / 2\right) g_{2}\left(y_{j}\right)\right] \exp \left[\mathrm{i} \frac{B}{2} \sum_{i=1}^{m-2}\left\langle\sum_{j=1}^{i} y_{j} \mid \mathrm{J} y_{i+1}\right\rangle\right] .
$$


Now we insert the scaling parameter $L>0$ and apply Roccaforte's asymptotic expansion of Appendix A up to the first order

$$
\begin{aligned}
\int_{\mathbb{R}^{2}} \mathrm{~d} x 1_{L \Lambda}(x) 1_{L \Lambda}\left(x-y_{1}\right) \cdots 1_{L \Lambda}\left(x-y_{m}\right) \\
\quad=\left|L \Lambda \cap\left(y_{1}+L \Lambda\right) \cap\left(y_{1}+y_{2}+L \Lambda\right) \cap \cdots \cap\left(y_{m}+L \Lambda\right)\right| \\
=|L \Lambda|-\left|L \Lambda \backslash\left(L \Lambda \cap\left(y_{1}+L \Lambda\right) \cap\left(y_{1}+y_{2}+L \Lambda\right) \cap \cdots \cap\left(y_{m}+L \Lambda\right)\right)\right| \\
\quad=L^{2}|\Lambda|-L \int_{\partial \Lambda} \mathrm{dA}(x) \max \left\{0,\left\langle y_{1} \mid n_{x}\right\rangle,\left\langle y_{1}+y_{2} \mid n_{x}\right\rangle, \ldots,\left\langle y_{m} \mid n_{x}\right\rangle\right\}+W\left(\sum_{j=1}^{m}\left\|y_{j}\right\|^{2}\right),
\end{aligned}
$$

as $L \rightarrow \infty$. Here, $A$ is the canonical arc-length measure on $\partial \Lambda$ and $n_{x}$ is the inward unit normal vector at the point $x \in \partial \Lambda$ and $W$ is a linearly bounded function on the positive half-line. We scale $\mathbf{y}$ by $B^{-1 / 2}$ and $\Lambda$ by $B^{1 / 2}$. Then we can set from now on $B=1$ in the function $\mathfrak{f}_{m}$ and replace $L \Lambda$ by $\sqrt{L^{2} B} \Lambda$. The parameter that tends to infinity in our asymptotic analysis is thus effectively $\sqrt{L^{2} B}$.

For a given point on the boundary curve, $x \in \partial \Lambda$, we decompose each vector $y_{i} \in \mathbb{R}^{2}$ into a component parallel and a component perpendicular to the tangent (line) $\mathrm{T}_{x}(\partial \Lambda) \cong$ $\mathbb{R}$ at $x \in \partial \Lambda$ according to

$$
y_{i}=-z_{i} \mathrm{~J} n_{x}+t_{i} n_{x}, \quad i=1, \ldots, m-1,
$$

with the real numbers $t_{i}:=\left\langle y_{i} \mid n_{x}\right\rangle$ and $z_{i}:=-\left\langle y_{i} \mid \mathrm{J} n_{x}\right\rangle$ so that $\left\|y_{i}\right\|^{2}=z_{i}^{2}+t_{i}^{2}$. Then we get

$$
\mathcal{L}_{\ell}\left(\left\|y_{i}\right\|^{2} / 2\right) g_{2}\left(y_{i}\right)=\mathcal{L}_{\ell}\left(\left(z_{i}^{2}+t_{i}^{2}\right) / 2\right) g\left(z_{i}\right) g\left(t_{i}\right)
$$

and

$$
\sum_{i=1}^{m-2}\left\langle\sum_{j=1}^{i} y_{j} \mid \mathrm{J} y_{i+1}\right\rangle=\sum_{i=1}^{m-1} z_{i} \sum_{j=1}^{m-1} \mathrm{~S}_{i j} t_{j}=\langle\mathbf{z} \mid \mathbf{S} \mathbf{t}\rangle
$$

to be used on the right-hand side of (3.6). Here, $\mathbf{z}:=\left(z_{1}, \ldots, z_{m-1}\right)$ and $\mathbf{t}:=$ $\left(t_{1}, \ldots, t_{m-1}\right)$. Moreover, $\mathrm{S}$ is the $(m-1) \times(m-1)$ matrix with entries

$$
\mathrm{S}_{i j}:=\left\{\begin{array}{cl}
-1 & \text { if } i<j \\
0 & \text { if } i=j \\
1 & \text { if } i>j
\end{array} .\right.
$$

By setting $t_{0}:=0$ the maximum in (3.6) can now be written as follows

$$
\max \left\{0,\left\langle y_{1} \mid n_{x}\right\rangle,\left\langle y_{1}+y_{2} \mid n_{x}\right\rangle, \ldots,\left\langle y_{1}+\cdots+y_{m-1} \mid n_{x}\right\rangle\right\}=\max _{0 \leq q \leq m-1} \sum_{r=0}^{q} t_{r}=: M(\mathbf{t}) .
$$

Let us now introduce new variables $\left(T_{1}, \ldots, T_{m-1}\right)$ by the sums

$$
T_{i}:=\sum_{j=1}^{m-1} \mathrm{~S}_{i j} t_{j}
$$

We also define $T_{m}:=0, t_{m}:=t_{1}+\cdots+t_{m-1}$, and $z_{m}:=z_{1}+\cdots+z_{m-1}$. 
The change (3.8) from the (global) variables $y_{i}$ to the $x$-dependent (local) variables $\left(z_{i}, t_{i}\right)$ corresponds to a translation and a rotation of the coordinate system. This implies that $\mathrm{d} y_{i}=\mathrm{d} t_{i} \mathrm{~d} z_{i}$ which is shorthand for the underlying invariance of the multidimensional Lebesgue measure. Once the integration with respect to all the variables $z_{i}$ and $t_{i}$ is done, the result will turn out to be independent of $x \in \partial \Lambda$ and the remaining integration with respect to $x$ along the boundary curve $\partial \Lambda$ simply yields the factor $L \sqrt{B}|\partial \Lambda|$.

By combining (3.5), (3.6), (3.8), (3.9), (3.10), and (3.12), and by referring to the Fubini-Tonelli theorem we get for the time being

$$
\begin{aligned}
& \operatorname{tr} \mathrm{P}_{\ell}(\Lambda)^{m}=\int_{\mathbb{R}^{m-1}} \mathrm{dt} g\left(t_{1}\right) \cdots g\left(t_{m-1}\right) g\left(t_{m}\right) I_{m}(\mathbf{t}) \\
& \quad \times\left(L^{2} B|\Lambda|-L \sqrt{B} \int_{\partial \Lambda} \mathrm{dA}(x) M(\mathbf{t})\right)+\mathcal{O}(1),
\end{aligned}
$$

with

$$
I_{m}(\mathbf{t}):=\int_{\mathbb{R}^{m-1}} \mathrm{~d} \mathbf{z} \prod_{j=1}^{m} g_{T_{j}, t_{j}}\left(z_{j}\right)
$$

and

$$
g_{T, t}(z):=\mathcal{L}_{\ell}\left(\left(z^{2}+t^{2}\right) / 2\right) g(z) \exp \left(\frac{\mathrm{i}}{2} T z\right), \quad T, t, z \in \mathbb{R}
$$

[When it comes to integration we do not switch from the $t$-variables to the $T$-variables; moreover, we note that $\operatorname{det} S=0$, resp. $=1$ if $m$ is even, resp. odd.] The integral $I_{m}(\mathbf{t})$ can be viewed as the $m$-fold convolution product $g_{T_{1}, t_{1}} * \cdots * g_{T_{m}, t_{m}}$ evaluated at 0 . This suggests to introduce the (inverse) Fourier transform

$$
\begin{gathered}
\check{g}_{T, t}(\xi):=\frac{1}{\sqrt{2 \pi}} \int_{\mathbb{R}} \mathrm{d} \omega g_{T, t}(\omega) \exp (\mathrm{i} \omega \xi) \\
=\frac{1}{2 \pi} \int_{\mathbb{R}} \mathrm{d} \omega \mathcal{L}_{\ell}\left(\left(\omega^{2}+t^{2}\right) / 2\right) \exp \left(-\omega^{2} / 4+\mathrm{i} T \omega / 2+\mathrm{i} \omega \xi\right) \\
\left.=\frac{1}{2 \pi} \exp \left[-(\xi+T / 2)^{2}\right] \int_{\mathbb{R}} \mathrm{d} \omega \mathcal{L}_{\ell}\left((\omega+\mathrm{i}(T+2 \xi))^{2}+t^{2}\right) / 2\right) \exp \left(-\omega^{2} / 4\right) .
\end{gathered}
$$

If $\ell=0$, then this integral can be calculated explicitly. But even then it turns out to be more convenient not to perform this integration at this point.

Therefore, the $(m-1)$-fold integral (3.15) can be rewritten as an integral over the real line according to

$$
I_{m}(\mathbf{t})=(2 \pi)^{m / 2} \int_{\mathbb{R}} \frac{\mathrm{d} \xi}{2 \pi} \prod_{j=1}^{m} \breve{g}_{T_{j}, t_{j}}(\xi)
$$


and the term of the sub-leading order $L$ in (3.14) becomes equal to (using the notation $\left.\omega:=\left(\omega_{1}, \ldots, \omega_{m}\right)\right)$

$$
\begin{aligned}
- & L \sqrt{B}|\partial \Lambda| \int_{\mathbb{R}^{m-1}} \mathrm{~d} \mathbf{t} M(\mathbf{t}) g\left(t_{1}\right) \cdots g\left(t_{m-1}\right) g\left(t_{m}\right) I_{m}(\mathbf{t}) \\
& =-L \sqrt{B}|\partial \Lambda|(2 \pi)^{-m / 2} \int_{\mathbb{R}^{m}} \mathrm{~d} \boldsymbol{\omega} \int_{\mathbb{R}} \frac{\mathrm{d} \xi}{2 \pi} \int_{\mathbb{R}^{m-1}} \mathrm{~d} \mathbf{t} M(\mathbf{t}) \\
& \left.\times \prod_{j=1}^{m} g\left(t_{j}\right) \exp \left(-\left(\xi+T_{j} / 2\right)^{2}\right) \mathcal{L}_{\ell}\left(\left(\omega_{j}+\mathrm{i}\left(T_{j}+2 \xi\right)\right)^{2}+t_{j}^{2}\right) / 2\right) \exp \left(-\omega_{j}^{2} / 4\right) .
\end{aligned}
$$

Following Roccaforte [25] we now introduce $m(\geq 2)$ subsets $\mathcal{S}, \mathcal{S}_{1}, \ldots, \mathcal{S}_{(m-1)}$ of $\mathbb{R}^{m-1}$ by

$$
\mathcal{S}:=\left\{\mathbf{t} \in \mathbb{R}^{m-1}: M(\mathbf{t})>0\right\}
$$

and

$\mathcal{S}_{q}:=\left\{\mathbf{t} \in \mathbb{R}^{m-1}: \sum_{r=s}^{q} t_{r}>0\right.$ for $1 \leq s \leq q$ and $\sum_{r=q+1}^{q+p} t_{r}<0$ for $\left.1 \leq p \leq m-1-q\right\}$

for $1 \leq q \leq m-1$. The sets $\mathcal{S}_{q}$ are pairwise disjoint and make up all of $\mathcal{S}$ in the sense that $\mathcal{S}=\bigcup_{q=1}^{m-1} \mathcal{S}_{q}$, up to (hyperplane) sets of ( $\left.m-1\right)$-dimensional Lebesgue measure zero. In fact, $\mathbf{t} \in \mathcal{S}_{q}$ implies that $M(\mathbf{t})=\sum_{r=1}^{q} t_{r}>0$. And the conditions for $2 \leq s \leq q$ and for $1 \leq p \leq m-1-q$ ensure that the sets $\mathcal{S}_{q}$ are indeed disjoint. Following again Roccaforte [25] we introduce variables $\tau:=\left(\tau_{1}, \ldots, \tau_{m-1}\right)$ adapted to the just introduced sets. We define

$$
\tau_{s}:=\sum_{r=s}^{q} t_{r}
$$

for $1 \leq s \leq q$ and

$$
\tau_{q+p}:=\sum_{r=q+1}^{q+p} t_{r}
$$

for $1 \leq p \leq m-1-q$.

On the set $\mathcal{S}_{q}$ we have $\boldsymbol{\tau}^{\prime}=\mathrm{A}^{(q)} \mathbf{t}^{\prime}$. Here $\boldsymbol{\tau}^{\prime}$ denotes the column tuple as the transpose of the (row) tuple $\boldsymbol{\tau}$ and similarly for $\mathbf{t}^{\prime}$. And the $(m-1) \times(m-1)$ matrix $\mathbf{A}^{(q)}$ is defined in terms of its entries

$$
\mathrm{A}^{(q)}(i, j):= \begin{cases}1 & \text { if } 1 \leq i \leq j \leq q \\ 1 & \text { if } q+1 \leq j \leq i \\ 0 & \text { otherwise }\end{cases}
$$

Then we have $\operatorname{det} \mathrm{A}^{(q)}=1$ and on $\mathcal{S}_{q}$ the comforting identity

$$
M(\mathbf{t})=\tau_{1} 1_{+}\left(\tau_{1}\right) \cdots 1_{+}\left(\tau_{q}\right) 1_{-}\left(\tau_{q+1}\right) \cdots 1_{-}\left(\tau_{m-1}\right), \quad \mathbf{t} \in \mathcal{S}_{q},
$$

using the abbreviations $1_{ \pm}$for the indicator functions on the real line $\mathbb{R}$ for its two half-lines $\mathbb{R}_{ \pm}$. 
Now we consider the joint integration with respect to the $m$ variables $\xi$ and $\tau$ and apply the following changes of variables. Firstly, we change $\tau_{q+1}, \ldots, \tau_{m-1}$ to $-\tau_{q+1}, \ldots,-\tau_{m-1}$. Clearly, the $\tau$ integral is now over $\mathbb{R}_{+}^{m-1}$. Secondly, we replace $\xi$ by $\xi-\left(\tau_{1}+\tau_{m-1}\right) / 2$, and thirdly we replace $\xi$ by $-\xi$. The negative of the argument in the product of exponentials in (3.20) then changes according to

$$
m \xi^{2}+\xi \sum_{i=1}^{m} T_{i}+\frac{1}{4} \sum_{i=1}^{m} T_{i}^{2}+\frac{1}{4} \sum_{i=1}^{m} t_{i}^{2} \rightsquigarrow \xi^{2}+\left(\xi+\tau_{1}\right)^{2}+\cdots+\left(\xi+\tau_{m-1}\right)^{2} .
$$

Here and in the following we are using the notation $\rightsquigarrow$ to present the results of changes of variables efficiently, without the explicit introduction of the underlying mappings. We prove (3.26) in Appendix B.1. The main advantage of the quadratic form (3.26) over that in the t-variables is that there are no mixed terms between the $\tau$ 's and the exponential can be factorized. The resulting term does not depend on $q$. This turns out to remain true with the Laguerre polynomials included as we will see next.

We perform the same changes of variables in the arguments of the Laguerre polynomials. For instance, if $q=1$, then

$$
\begin{aligned}
\left(\omega+\mathrm{i}\left(2 \xi+T_{1}\right)\right)^{2}+t_{1}^{2} & =\left(\omega+\mathrm{i}\left(2 \xi-\tau_{m-1}\right)\right)^{2}+\tau_{1}^{2} \\
& \rightsquigarrow\left(\omega-\mathrm{i}\left(2 \xi+\tau_{1}\right)\right)^{2}+\tau_{1}^{2} \\
& =\omega^{2}-2 \mathrm{i} \omega\left(2 \xi+\tau_{1}\right)-(2 \xi)^{2}-4 \xi \tau_{1} .
\end{aligned}
$$

Next we change $\tau_{1}$ to $\tau_{1}-\xi$ so that the last expression equals

$$
\omega^{2}-2 \mathrm{i} \omega\left(\xi+\tau_{1}\right)-4 \xi \tau_{1}=(\omega-2 \mathrm{i} \xi)\left(\omega-2 \mathrm{i} \tau_{1}\right) .
$$

Similarly,

$$
\begin{gathered}
\left(\omega+\mathrm{i}\left(2 \xi+T_{2}\right)\right)^{2}+t_{2}^{2} \rightsquigarrow(\omega-2 \mathrm{i} \xi)\left(\omega-2 \mathrm{i} \tau_{2}\right), \\
\left(\omega+\mathrm{i}\left(2 \xi+T_{3}\right)\right)^{2}+t_{3}^{2} \rightsquigarrow\left(\omega-2 \mathrm{i} \tau_{2}\right)\left(\omega-2 \mathrm{i} \tau_{3}\right), \\
\left(\omega+\mathrm{i}\left(2 \xi+T_{4}\right)\right)^{2}+t_{4}^{2} \rightsquigarrow\left(\omega-2 \mathrm{i} \tau_{3}\right)\left(\omega-2 \mathrm{i} \tau_{4}\right), \\
:: \\
\left(\omega+\mathrm{i}\left(2 \xi+T_{m-1}\right)\right)^{2}+t_{m-1}^{2} \rightsquigarrow\left(\omega-2 \mathrm{i} \tau_{m-2}\right)\left(\omega-2 \mathrm{i} \tau_{m-1}\right), \\
\left(\omega+\mathrm{i}\left(2 \xi+T_{m}\right)\right)^{2}+t_{m}^{2} \rightsquigarrow\left(\omega-2 \mathrm{i} \tau_{m-1}\right)\left(\omega-2 \mathrm{i} \tau_{1}\right) .
\end{gathered}
$$

For general $q \geq 2$, see Appendix B.2. In the end, the product of the Laguerre polynomials equals

$$
\begin{aligned}
& \quad \prod_{1 \leq j \leq q-1} \mathcal{L}_{\ell}\left(\left(\omega_{j}-2 \mathrm{i} \tau_{j}\right)\left(\omega_{j}-2 \mathrm{i} \tau_{j+1}\right) / 2\right) \prod_{q+2 \leq j \leq m} \mathcal{L}_{\ell}\left(\left(\omega_{j}-2 \mathrm{i} \tau_{j-1}\right)\left(\omega_{j}-2 \mathrm{i} \tau_{j}\right) / 2\right) \\
& \quad \times \mathcal{L}_{\ell}\left(\left(\omega_{q}-2 \mathrm{i} \xi\right)\left(\omega_{q}-2 \mathrm{i} \tau_{q}\right) / 2\right) \mathcal{L}_{\ell}\left(\left(\omega_{q+1}-2 \mathrm{i} \xi\right)\left(\omega_{q+1}-2 \mathrm{i} \tau_{q+1}\right) / 2\right) \\
& \quad \times \mathcal{L}_{\ell}\left(\left(\omega_{q}-2 \mathrm{i} \tau_{1}\right)\left(\omega_{q}-2 \mathrm{i} \tau_{m-1}\right) / 2\right) .
\end{aligned}
$$

The following remarkable identity will be proved in Appendix B.3,

$$
\frac{1}{\sqrt{2 \pi}} \int_{\mathbb{R}} \mathrm{d} \omega \mathcal{L}_{\ell}((\omega-2 \mathrm{i} \xi)(\omega-2 \mathrm{i} \tau) / 2) \exp \left(-\omega^{2} / 4\right)=\sqrt{2}\left(2^{\ell} \ell !\right)^{-1} H_{\ell}(\xi) H_{\ell}(\tau) .
$$


After performing the $m$-fold integration with respect to $\omega$ we obtain

$$
2^{m / 2}\left(2^{\ell} \ell !\right)^{-1} H_{\ell}^{2}(\xi)\left(2^{\ell} \ell !\right)^{-1} H_{\ell}^{2}\left(\tau_{1}\right) \cdots\left(2^{\ell} \ell !\right)^{-1} H_{\ell}^{2}\left(\tau_{m-1}\right) .
$$

To summarize, the boundary-curve term of the order $L$ equals $-L \sqrt{B}|\partial \Lambda| /(2 \pi)$ times

$$
\begin{aligned}
(m-1)\left(\sqrt{\pi} 2^{\ell} \ell !\right)^{-1} \int_{\mathbb{R}} \mathrm{d} \xi & H_{\ell}^{2}(\xi) \exp \left(-\xi^{2}\right) \int_{\xi}^{\infty} \mathrm{d} \tau_{1}\left(\tau_{1}-\xi\right) H_{\ell}^{2}\left(\tau_{1}\right) \exp \left(-\tau_{1}^{2}\right) \lambda_{\ell}(\xi)^{m-2} \\
= & -\int_{\mathbb{R}} \mathrm{d} \xi(m-1) \lambda_{\ell}^{\prime}(\xi) \lambda(\xi)^{m-2} \int_{\xi}^{\infty} \mathrm{d} \tau(\tau-\xi) H_{\ell}^{2}(\tau) \exp \left(-\tau^{2}\right) \\
= & -\int_{\mathbb{R}} \mathrm{d} \xi \frac{\mathrm{d}}{\mathrm{d} \xi}\left(\lambda_{\ell}(\xi)^{m-1}-1\right) \int_{\xi}^{\infty} \mathrm{d} \tau(\tau-\xi) H_{\ell}^{2}(\tau) \exp \left(-\tau^{2}\right) \\
= & -\left[\left(\lambda_{\ell}(\xi)^{m-1}-1\right) \int_{\xi}^{\infty} \mathrm{d} \tau(\tau-\xi) H_{\ell}^{2}(\tau) \exp \left(-\tau^{2}\right)\right]_{-\infty}^{\infty} \\
& +\int_{\mathbb{R}} \mathrm{d} \xi\left(\lambda_{\ell}(\xi)^{m-1}-1\right) \frac{\mathrm{d}}{\mathrm{d} \xi} \int_{\xi}^{\infty} \mathrm{d} \tau(\tau-\xi) H_{\ell}^{2}(\tau) \exp \left(-\tau^{2}\right) \\
= & -\int_{\mathbb{R}} \mathrm{d} \xi\left(\lambda_{\ell}(\xi)^{m-1}-1\right) \lambda_{\ell}(\xi)
\end{aligned}
$$

Finally, we turn to the leading area term of the order $L^{2}$ in (3.14),

$$
L^{2} B|\Lambda| \int_{\mathbb{R}^{m-1}} \mathrm{dt} g\left(t_{1}\right) \cdots g\left(t_{m-1}\right) g\left(t_{m}\right) I_{m}(\mathbf{t}) .
$$

Here, we use (3.19) for $I_{m}(\mathbf{t})$ and switch to the variables $\tau_{1}, \ldots, \tau_{m-1}$ from (3.23) and (3.24) for $q=1$, in all of $\mathbb{R}^{m-1}$. We perform the same shifts in $\xi$ and in the $\tau$ 's. Then the area term turns into

$$
L^{2} B \frac{|\Lambda|}{2 \pi}\left(\int_{\mathbb{R}} \mathrm{d} \xi\left(\sqrt{\pi} 2^{\ell} \ell !\right)^{-1} H_{\ell}^{2}(\xi) \exp \left(-\xi^{2}\right)\right)^{m}=L^{2} B \frac{|\Lambda|}{2 \pi}
$$

by the normalization of the Hermite functions. Alternatively, the leading term can be obtained by replacing the $x$-integral in (3.5) by $|\Lambda|$. The remaining $\mathbf{y}$-integration yields $B /(2 \pi)$. This finishes the proof of Lemma 5 .

The next lemma provides the basis for the proof of Theorem 2 .

Lemma 6. Under the same assumptions as in Lemma 5 we have for any $n \in \mathbb{N}_{0}$

$$
\operatorname{tr} \mathrm{P}_{\leq n}(L \Lambda)^{m}=L^{2} B \frac{|\Lambda|}{2 \pi}(n+1)+L \sqrt{B}|\partial \Lambda| \int_{\mathbb{R}} \frac{\mathrm{d} \xi}{2 \pi}\left[\operatorname{tr} \mathcal{K}_{n, \xi}^{m}-\operatorname{tr} \mathcal{K}_{n, \xi}\right]+\mathcal{O}(1)
$$

as $L \rightarrow \infty$.

Proof. By the same arguments as in the beginning of the proof of Lemma 5, the projection $\mathrm{P}_{\leq n}(\Lambda)^{m}$ is a trace-class operator and its trace can be calculated by integrating the diagonal of the $m$-fold iterated integral kernel of $\mathrm{P}_{\leq n}(\Lambda)$. Again, the case $m=1$ is then obvious and we only need to consider the case $m \geq 2$. We recall that $\mathrm{P}_{\leq n}(x, y)=$ 
$\sum_{0 \leq \ell \leq n} p_{\ell}(x, y)$. So in the proof of Lemma 5 we simply have to replace $\mathcal{L}_{\ell}$ with $\mathcal{L}_{\leq n}=$ $\sum_{0 \leq \ell \leq n} \mathcal{L}_{\ell}=\mathcal{L}_{n}^{(1)}$. For instance, expression (3.27) is replaced with the expression

$$
\begin{aligned}
\prod_{1 \leq j \leq q-1} & \mathcal{L}_{\leq n}\left(\left(\omega_{j}-2 \mathrm{i} \tau_{j}\right)\left(\omega_{j}-2 \mathrm{i} \tau_{j+1}\right) / 2\right) \prod_{q+2 \leq j \leq m} \mathcal{L}_{\leq n}\left(\left(\omega_{j}-2 \mathrm{i} \tau_{j-1}\right)\left(\omega_{j}-2 \mathrm{i} \tau_{j}\right) / 2\right) \\
& \times \mathcal{L}_{\leq n}\left(\left(\omega_{q}-2 \mathrm{i} \xi\right)\left(\omega_{q}-2 \mathrm{i} \tau_{q}\right) / 2\right) \mathcal{L}_{\leq n}\left(\left(\omega_{q+1}-2 \mathrm{i} \xi\right)\left(\omega_{q+1}-2 \mathrm{i} \tau_{q+1}\right) / 2\right) \\
& \times \mathcal{L}_{\leq n}\left(\left(\omega_{q}-2 \mathrm{i} \tau_{1}\right)\left(\omega_{q}-2 \mathrm{i} \tau_{m-1}\right) / 2\right)
\end{aligned}
$$

We multiply this expression by $(2 \pi)^{-m / 2} \prod_{j=1}^{m} \exp \left(-\omega_{j}{ }^{2} / 4\right)$ and integrate with respect to $\omega$ over $\mathbb{R}^{m}$ by using (3.28). This yields

$$
\begin{aligned}
2^{m / 2} & \prod_{1 \leq j \leq q-1} \sum_{\ell_{j}=0}^{n}\left(2^{\ell_{j}} \ell_{j} !\right)^{-1} H_{\ell_{j}}\left(\tau_{j}\right) H_{\ell_{j}}\left(\tau_{j+1}\right) \prod_{q+2 \leq j \leq m-1} \sum_{\ell_{j}=0}^{n}\left(2^{\ell_{j}} \ell_{j} !\right)^{-1} H_{\ell_{j}}\left(\tau_{j-1}\right) H_{\ell_{j}}\left(\tau_{j}\right) \\
& \times \sum_{\ell_{q}=0}^{n}\left(2^{\ell_{q}} \ell_{q} !\right)^{-1} H_{\ell_{q}}(\xi) H_{\ell_{q}}\left(\tau_{q}\right) \sum_{\ell_{q+1}=0}^{n}\left(2^{\ell_{q+1}} \ell_{q+1} !\right)^{-1} H_{\ell_{q+1}}(\xi) H_{\ell_{q+1}}\left(\tau_{q+1}\right) \\
& \times \sum_{\ell_{m}=0}^{n}\left(2^{\ell_{m}} \ell_{m} !\right)^{-1} H_{\ell_{m}}\left(\tau_{1}\right) H_{\ell_{m}}\left(\tau_{m-1}\right)
\end{aligned}
$$

To pause for a moment, the term of the order $L$ equals $-\sqrt{B} L|\partial \Lambda| /(2 \pi)$ times

$$
\begin{aligned}
& \sum_{q=1}^{m-1} \int_{\mathbb{R}} \frac{\mathrm{d} \xi}{2 \pi} \exp \left(-\xi^{2}\right) \int_{\xi}^{\infty} \mathrm{d} \tau_{1}\left(\tau_{1}-\xi\right) \exp \left(-\tau_{1}^{2}\right) \int_{\xi}^{\infty} \mathrm{d} \tau_{2} \exp \left(-\tau_{2}^{2}\right) \cdots \int_{\xi}^{\infty} \mathrm{d} \tau_{m-1} \exp \left(-\tau_{m-1}^{2}\right) \\
& \quad \times \pi^{-m / 2} \prod_{1 \leq j \leq q-1} \sum_{\ell_{j}=0}^{n}\left(2^{\ell_{j}} \ell_{j} !\right)^{-1} H_{\ell_{j}}\left(\tau_{j}\right) H_{\ell_{j}}\left(\tau_{j+1}\right) \prod_{q+2 \leq j \leq m-1} \sum_{\ell_{j}=0}^{n}\left(2^{\ell_{j}} \ell_{j} !\right)^{-1} H_{\ell_{j}}\left(\tau_{j-1}\right) H_{\ell_{j}}\left(\tau_{j}\right) \\
& \quad \times \sum_{\ell_{q}=0}^{n}\left(2^{\ell_{q}} \ell_{q} !\right)^{-1} H_{\ell_{q}}(\xi) H_{\ell_{q}}\left(\tau_{q}\right) \sum_{\ell_{q+1}=0}^{n}\left(2^{\ell_{q+1}} \ell_{q+1} !\right)^{-1} H_{\ell_{q+1}}(\xi) H_{\ell_{q+1}}\left(\tau_{q+1}\right) \\
& \quad \times \sum_{\ell_{m}=0}^{n}\left(2^{\ell_{m}} \ell_{m} !\right)^{-1} H_{\ell_{m}}\left(\tau_{1}\right) H_{\ell_{m}}\left(\tau_{m-1}\right) .
\end{aligned}
$$

We include the factors of $\pi$ into the terms $\left(2^{\ell} \ell !\right)^{-1 / 2}$, split them in halves, and combine them with each corresponding factor $H_{\ell}$. In accordance with that we define

$$
\lambda_{\ell_{i}, \ell_{j}}(\xi):=\int_{\xi}^{\infty} \mathrm{d} \tau\left(\sqrt{\pi} 2^{\ell_{i}} \ell_{i} !\right)^{-1 / 2} H_{\ell_{i}}(\tau)\left(\sqrt{\pi} 2^{\ell_{j}} \ell_{j} !\right)^{-1 / 2} H_{\ell_{j}}(\tau) \exp \left(-\tau^{2}\right) .
$$

Then the summand for $q=1$ can be written in the form

$$
\begin{aligned}
-\sum_{0 \leq \ell_{1}, \ldots, \ell_{m} \leq n} & \int_{\mathbb{R}} \frac{\mathrm{d} \xi}{2 \pi}\left[\frac{\mathrm{d}}{\mathrm{d} \xi} \lambda_{\ell_{1}, \ell_{2}}(\xi)\right] \lambda_{\ell_{2}, \ell_{3}}(\xi) \cdots \lambda_{\ell_{m-1}, \ell_{m}}(\xi) \\
& \times \int_{\xi}^{\infty} \mathrm{d} \tau_{1}\left(\tau_{1}-\xi\right)\left(\sqrt{\pi} 2^{\ell_{1}} \ell_{1} !\right)^{-1 / 2} H_{\ell_{1}}\left(\tau_{1}\right)\left(\sqrt{\pi} 2^{\ell_{m}} \ell_{m} !\right)^{-1 / 2} H_{\ell_{m}}\left(\tau_{1}\right) \exp \left(-\tau_{1}^{2}\right) .
\end{aligned}
$$

For $q=2$ we get the term

$$
\begin{aligned}
-\sum_{0 \leq \ell_{1}, \ldots, \ell_{m} \leq n} & \int_{\mathbb{R}} \frac{\mathrm{d} \xi}{2 \pi} \lambda_{\ell_{1}, \ell_{2}}(\xi)\left[\frac{\mathrm{d}}{\mathrm{d} \xi} \lambda_{\ell_{2}, \ell_{3}}(\xi)\right] \lambda_{\ell_{3}, \ell_{4}}(\xi) \cdots \lambda_{\ell_{m-1}, \ell_{m}}(\xi) \\
& \times \int_{\xi}^{\infty} \mathrm{d} \tau_{1}\left(\tau_{1}-\xi\right)\left(\sqrt{\pi} 2^{\ell_{1}} \ell_{1} !\right)^{-1 / 2} H_{\ell_{1}}\left(\tau_{1}\right)\left(\sqrt{\pi} 2^{\ell_{m}} \ell_{m} !\right)^{-1 / 2} H_{\ell_{m}}\left(\tau_{1}\right) \exp \left(-\tau_{1}^{2}\right),
\end{aligned}
$$


and similarly for $q=3, \ldots, m-1$. By summing over all $q$ we obtain

$$
\begin{aligned}
-\sum_{0 \leq \ell_{1}, \ldots, \ell_{m} \leq n} & \int_{\mathbb{R}} \frac{\mathrm{d} \xi}{2 \pi} \frac{\mathrm{d}}{\mathrm{d} \xi}\left[\lambda_{\ell_{1}, \ell_{2}}(\xi) \lambda_{\ell_{2}, \ell_{3}}(\xi) \cdots \lambda_{\ell_{m-1}, \ell_{m}}(\xi)\right] \\
& \times \int_{\xi}^{\infty} \mathrm{d} \tau_{1}\left(\tau_{1}-\xi\right)\left(\sqrt{\pi} 2^{\ell_{1}} \ell_{1} !\right)^{-1 / 2} H_{\ell_{1}}\left(\tau_{1}\right)\left(\sqrt{\pi} 2^{\ell_{m}} \ell_{m} !\right)^{-1 / 2} H_{\ell_{m}}\left(\tau_{1}\right) \exp \left(-\tau_{1}^{2}\right) .
\end{aligned}
$$

Inside the derivative with respect to $\xi$ we subtract the constant $C_{\ell_{1}, \ldots, \ell_{m}}:=$ $\lambda_{\ell_{1}, \ell_{2}}(-\infty) \lambda_{\ell_{2}, \ell_{3}}(-\infty) \cdots \lambda_{\ell_{m-1}, \ell_{m}}(-\infty)$ so that we can integrate by parts. Then we get

$$
\begin{aligned}
-\int_{\mathbb{R}} \frac{\mathrm{d} \xi}{2 \pi} & \frac{\mathrm{d}}{\mathrm{d} \xi}\left[\lambda_{\ell_{1}, \ell_{2}}(\xi) \lambda_{\ell_{2}, \ell_{3}}(\xi) \cdots \lambda_{\ell_{m-1}, \ell_{m}}(\xi)-C_{\ell_{1}, \ldots, \ell_{m}}\right] \\
& \times \int_{\xi}^{\infty} \mathrm{d} \tau_{1}\left(\tau_{1}-\xi\right)\left(\sqrt{\pi} 2^{\ell_{1}} \ell_{1} !\right)^{-1 / 2} H_{\ell_{1}}\left(\tau_{1}\right)\left(\sqrt{\pi} 2^{\ell_{m}} \ell_{m} !\right)^{-1 / 2} H_{\ell_{m}}\left(\tau_{1}\right) \exp \left(-\tau_{1}^{2}\right) \\
& =-\int_{\mathbb{R}} \frac{\mathrm{d} \xi}{2 \pi}\left[\lambda_{\ell_{1}, \ell_{2}}(\xi) \lambda_{\ell_{2}, \ell_{3}}(\xi) \cdots \lambda_{\ell_{m-1}, \ell_{m}}(\xi)-C_{\ell_{1}, \ldots, \ell_{m}}\right] \lambda_{\ell_{1}, \ell_{m}}(\xi) \\
& =-\int_{\mathbb{R}} \frac{\mathrm{d} \xi}{2 \pi}\left[\lambda_{\ell_{1}, \ell_{2}}(\xi) \lambda_{\ell_{2}, \ell_{3}}(\xi) \cdots \lambda_{\ell_{m-1}, \ell_{m}}(\xi) \lambda_{\ell_{m}, \ell_{1}}(\xi)-C_{\ell_{1}, \ldots, \ell_{m}} \lambda_{\ell_{1}, \ell_{m}}(\xi)\right] .
\end{aligned}
$$

By (2.7) we know that $C_{\ell_{1}, \ldots, \ell_{m}}=\delta_{\ell_{1}, \ell_{2}} \delta_{\ell_{2}, \ell_{3}} \cdots \delta_{\ell_{m-1}, \ell_{m}}$. Now we sum over $\ell_{1}, \ldots, \ell_{m}$ and use the Christoffel-Darboux formula (of the years 1858 and 1878)

$$
\sum_{\ell=0}^{n}\left(2^{\ell} \ell !\right)^{-1} H_{\ell}(\tau) H_{\ell}\left(\tau^{\prime}\right)=\left(2^{n+1} n !\right)^{-1} \frac{H_{n}\left(\tau^{\prime}\right) H_{n+1}(\tau)-H_{n}(\tau) H_{n+1}\left(\tau^{\prime}\right)}{\tau-\tau^{\prime}} \quad \text { if } \tau \neq \tau^{\prime}
$$

and

$$
\sum_{\ell=0}^{n}\left(2^{\ell} \ell !\right)^{-1} H_{\ell}(\tau)^{2}=\left(2^{n+1} n !\right)^{-1}\left[H_{n+1}^{2}(\tau)-H_{n}(\tau) H_{n+2}(\tau)\right] .
$$

Then, for instance,

$$
\begin{aligned}
\sum_{0 \leq \ell_{2} \leq n} \lambda_{\ell_{1}, \ell_{2}}(\xi) \lambda_{\ell_{2}, \ell_{3}}(\xi) \\
=\left(\sqrt{\pi} 2^{\ell_{1}} \ell_{1} !\right)^{-1 / 2}\left(\sqrt{\pi} 2^{\ell_{3}} \ell_{3} !\right)^{-1 / 2} \int_{[\xi, \infty)^{2}} \mathrm{~d} \tau_{1} \mathrm{~d} \tau_{2} H_{\ell_{1}}\left(\tau_{1}\right) H_{\ell_{3}}\left(\tau_{2}\right) \\
\quad \times \sum_{0 \leq \ell_{2} \leq n}\left(\sqrt{\pi} 2^{\ell_{2}} \ell_{2} !\right)^{-1} H_{\ell_{2}}\left(\tau_{1}\right) H_{\ell_{2}}\left(\tau_{2}\right) \exp \left(-\tau_{1}^{2}-\tau_{2}^{2}\right) \\
=\left(\sqrt{\pi} 2^{\ell_{1}} \ell_{1} !\right)^{-1 / 2}\left(\sqrt{\pi} 2^{\ell_{3}} \ell_{3} !\right)^{-1 / 2}\left(\sqrt{\pi} 2^{n+1} n !\right)^{-1} \\
\quad \times \int_{[\xi, \infty)^{2}} \mathrm{~d} \tau_{1} \mathrm{~d} \tau_{2} H_{\ell_{1}}\left(\tau_{1}\right) H_{\ell_{3}}\left(\tau_{2}\right) \frac{H_{n}\left(\tau_{2}\right) H_{n+1}\left(\tau_{1}\right)-H_{n}\left(\tau_{1}\right) H_{n+1}\left(\tau_{2}\right)}{\tau_{1}-\tau_{2}} \exp \left(-\tau_{1}^{2}-\tau_{2}^{2}\right) .
\end{aligned}
$$

Performing also the summations over $\ell_{1}, \ell_{3}, \ldots, \ell_{m}$ yields

$$
\begin{aligned}
\left(\sqrt{\pi} 2^{n+1} n !\right)^{-m} \int_{[\xi, \infty)^{m}} \mathrm{~d} \tau \exp \left(-\tau^{2}\right) \frac{H_{n}\left(\tau_{2}\right) H_{n+1}\left(\tau_{1}\right)-H_{n}\left(\tau_{1}\right) H_{n+1}\left(\tau_{2}\right)}{\tau_{1}-\tau_{2}} \\
\quad \times \frac{H_{n}\left(\tau_{3}\right) H_{n+1}\left(\tau_{2}\right)-H_{n}\left(\tau_{2}\right) H_{n+1}\left(\tau_{3}\right)}{\tau_{2}-\tau_{3}} \cdots \frac{H_{n}\left(\tau_{1}\right) H_{n+1}\left(\tau_{m}\right)-H_{n}\left(\tau_{m}\right) H_{n+1}\left(\tau_{1}\right)}{\tau_{m}-\tau_{1}} \\
=: \lambda_{\leq n, m}(\xi) .
\end{aligned}
$$


By (3.33) we also find

$$
\begin{aligned}
\sum_{0 \leq \ell_{1}, \ldots, \ell_{m} \leq n} C_{\ell_{1}, \ldots, \ell_{m}} \lambda_{\ell_{1}, \ell_{m}}(\xi) & =\sum_{0 \leq \ell \leq n}\left(\sqrt{\pi} 2^{\ell} \ell !\right)^{-1} \int_{\xi}^{\infty} \mathrm{d} \tau H_{\ell}(\tau)^{2} \exp \left(-\tau^{2}\right) \\
& =\left(\sqrt{\pi} 2^{n+1} n !\right)^{-1} \int_{\xi}^{\infty} \mathrm{d} \tau\left[H_{n+1}^{2}(\tau)-H_{n}(\tau) H_{n+2}(\tau)\right] \exp \left(-\tau^{2}\right) \\
& =: \lambda_{\leq n, 1}(\xi) .
\end{aligned}
$$

Now we apply (3.33) directly to the integral kernel of the operator $\mathcal{K}_{n, \xi}$ as defined in (2.11). Then we get

$$
\mathcal{K}_{n, \xi}\left(\tau, \tau^{\prime}\right)=\frac{\exp \left(-\left(\tau^{2}+\tau^{\prime 2}\right) / 2\right)}{\sqrt{\pi} 2^{n+1} n !} \begin{cases}\frac{H_{n}\left(\tau^{\prime}\right) H_{n+1}(\tau)-H_{n}(\tau) H_{n+1}\left(\tau^{\prime}\right)}{\tau-\tau^{\prime}} & \text { if } \tau \neq \tau^{\prime} \\ H_{n+1}^{2}(\tau)-H_{n}(\tau) H_{n+2}(\tau) & \text { if } \tau=\tau^{\prime}\end{cases}
$$

whenever $\tau \geq \xi$ and $\tau^{\prime} \geq \xi$ and zero otherwise. By comparing this with the just given definitions (3.34) and (3.35) of $\lambda_{\leq n, m}(\xi)$ we arrive at the relation $\lambda_{\leq n, m}(\xi)=\operatorname{tr} \mathcal{K}_{n, \xi}^{m}$ for all $m \in \mathbb{N}$ as claimed in Lemma 6 for the sub-leading term of the order $L$. Finally, we turn to the leading term of the order $L^{2}$. It equals

$$
\begin{aligned}
L^{2} B|\Lambda| & \int_{\mathbb{R}} \frac{\mathrm{d} \xi}{2 \pi} \exp \left(-\xi^{2}\right) \int_{\mathbb{R}} \mathrm{d} \tau_{1} \exp \left(-\tau_{1}^{2}\right) \cdots \int_{\mathbb{R}} \mathrm{d} \tau_{m-1} \exp \left(-\tau_{m-1}^{2}\right) \\
& \times \sum_{\ell_{1}=0}^{n}\left(\sqrt{\pi} 2^{\ell_{1}} \ell_{1} !\right)^{-1} H_{\ell_{1}}(\xi) H_{\ell_{1}}\left(\tau_{1}\right) \sum_{\ell_{2}=0}^{n}\left(\sqrt{\pi} 2^{\ell_{2}} \ell_{2} !\right)^{-1} H_{\ell_{2}}(\xi) H_{\ell_{2}}\left(\tau_{2}\right) \cdots \\
& \times \sum_{\ell_{m}=0}^{n}\left(\sqrt{\pi} 2^{\ell_{m}} \ell_{m} !\right)^{-1} H_{\ell_{m}}\left(\tau_{1}\right) H_{\ell_{m}}\left(\tau_{m-1}\right) .
\end{aligned}
$$

By the orthogonality (2.7) this yields $L^{2} B(n+1)|\Lambda| /(2 \pi)$ as claimed.

\section{From Smooth Functions to the Entropy Functions}

In this section we build on Theorems 1 and 2 with a suitable function $f$ to derive the precise leading asymptotic growth of the local ground-state entropy with arbitrary Rényi index $\alpha>0$. While the case $\alpha>1$ is rather straightforward, non-smoothness in the case $\alpha \leq 1$ requires considerable attention. In the first subsection we define the local groundstate entropies and present our main result and related results. The second subsection prepares the ground for getting from smooth functions to the non-smooth functions needed in the case $\alpha \leq 1$. Proofs of our results are then given in the third subsection.

4.1. Definitions and results. For a real parameter $\alpha>0$ we define the $\alpha$-Rényi entropy function $h_{\alpha}:[0,1] \rightarrow[0, \ln (2)]$ by

$$
\begin{aligned}
& h_{\alpha}(t):=\frac{1}{1-\alpha} \ln \left(t^{\alpha}+(1-t)^{\alpha}\right), \quad \alpha \neq 1, \\
& h_{1}(0):=h_{1}(1):=0, h_{1}(t):=-t \ln (t)-(1-t) \ln (1-t) \quad \text { if } t \notin\{0,1\}
\end{aligned}
$$


and recall (2.1) as well as (2.3) for the Landau Hamiltonian H.

Then the positive number

$$
S_{\alpha}(\Lambda):=\operatorname{tr} h_{\alpha}\left(\mathbb{1}_{\Lambda} \Theta(\mu \mathbb{1}-\mathrm{H}) \mathbb{1}_{\Lambda}\right)=\operatorname{tr} h_{\alpha}\left(\mathrm{P}_{\leq \nu}(\Lambda)\right)=\operatorname{tr} \mathbb{1}_{\Lambda} h_{\alpha}\left(\mathrm{P}_{\leq \nu}(\Lambda)\right) \mathbb{1}_{\Lambda}
$$

is the local $\alpha$-Rényi ground-state entropy (with chemical potential $\mu \geq B$ ), see (2.5) and [9]. Here, the integer $v \in \mathbb{N}_{0}$ is the integer part of $(\mu / B-1) / 2$ as defined already below (2.4). In particular, $S(\Lambda):=S_{1}(\Lambda)$ is the local von Neumann ground-state entropy mentioned in the abstract. The third equality in (4.2) is due to $h_{\alpha}(0)=0$.

Finiteness of the local $\alpha$-Rényi ground-state entropy is guaranteed by

Lemma 7. Let $\Lambda \subset \mathbb{R}^{2}$ be a bounded Borel set and $\mu \geq B$. Then $S_{\alpha}(\Lambda)<\infty$ for any $\alpha>0$.

The proof is given in subsection 4.3 after certain preparations in subsection 4.2. The next theorem gives the precise asymptotic growth. It is our main result.

Theorem 8 (Asymptotics of the local Rényi ground-state entropies). Let $\Lambda \subset \mathbb{R}^{2}$ be a bounded $\mathrm{C}^{3}$-region and let the chemical potential satisfy $\mu \geq B$. Then the local $\alpha$-Rényi ground-state entropy (4.2) obeys

$$
S_{\alpha}(L \Lambda)=L \sqrt{B}|\partial \Lambda| \mathrm{M}_{\leq v}\left(h_{\alpha}\right)+o(L),
$$

as $L \rightarrow \infty$. The asymptotic coefficient $\mathrm{M}_{\leq v}\left(h_{\alpha}\right)$ is given by (2.12) with $n=v$. It is finite and positive.

The proof is given in subsection 4.3. It builds on Lemma 7, Theorem 2, and Subsection 4.2.

Remarks 9. (i) The coefficient $\mathrm{M}_{\leq v}\left(h_{\alpha}\right)$ in (4.3) is in general not easy to calculate. The simplest case occurs when $v=0$. Then we have

$$
\mathrm{M}_{\leq 0}\left(h_{\alpha}\right)=\mathrm{M}_{0}\left(h_{\alpha}\right)=\int_{\mathbb{R}} \frac{\mathrm{d} \xi}{2 \pi} h_{\alpha}\left(\lambda_{0}(\xi)\right)
$$

with $\lambda_{0}(\xi)=\pi^{-1 / 2} \int_{\xi}^{\infty} \mathrm{d} t \exp \left(-t^{2}\right)$ being $1 / 2$ of the complementary error function. The coefficient (4.4) was found in [26] for $\alpha=1$ and special regions $\Lambda$. The first proof of (4.3) for $\alpha=1, v=0$ (equivalently, $\ell=0$ in (4.7)), $L^{2} B \in \mathbb{N}$, and general bounded $\mathrm{C}^{\infty}$-regions is due to Charles and Estienne in [4]. A numerical computation gives $\mathrm{M}_{0}\left(h_{1}\right)=0.203 \ldots$, also in agreement with [26].

(ii) In the zero-field case $B=0$, the leading term of the local Rényi ground-state entropy depends on its index $\alpha$ simply through the pre-factor $(1+\alpha) / \alpha$, see [16]. A numerical computation shows that in the case $B \neq 0$ the dependence on $\alpha$ is not so simple.

We are going to define a local $\alpha$-Rényi ground-state entropy also for the simpler situation where the Landau Hamiltonian $\mathrm{H}$ is restricted (or "projected") from the outset to a single Landau-level eigenspace $\mathrm{P}_{\ell} \mathrm{L}^{2}\left(\mathbb{R}^{2}\right)$ with arbitrary index $\ell \in \mathbb{N}_{0}$. This restriction means that $\mathrm{H}$ is replaced with $\mathrm{P}_{\ell} \mathrm{HP}_{\ell}$ and similarly for related operators, confer, for example, [11]. Then the corresponding local(alized) Fermi projection is in analogy to (2.5) given by

$\mathbb{1}_{\Lambda} \mathrm{P}_{\ell} \Theta\left(\mu \mathrm{P}_{\ell} \mathbb{1} \mathrm{P}_{\ell}-\mathrm{P}_{\ell} \mathrm{HP}_{\ell}\right) \mathrm{P}_{\ell} \mathbb{1}_{\Lambda}=\Theta(\mu-(2 \ell+1) B) \mathrm{P}_{\ell}(\Lambda)=\mathrm{P}_{\ell}(\Lambda), \quad \mu \geq(2 \ell+1) B$ 
We ignore the case $\mu<(2 \ell+1) B$, because then the local Fermi projection (4.5) is the zero operator. In analogy to (4.2) we now define for each $\ell \in \mathbb{N}_{0}$ the positive number

$$
S_{\alpha, \ell}(\Lambda):=\operatorname{tr} h_{\alpha}\left(\mathbb{1}_{\Lambda} \mathrm{P}_{\ell} \Theta\left(\mu \mathrm{P}_{\ell}-\mathrm{P}_{\ell} \mathrm{HP}_{\ell}\right) \mathrm{P}_{\ell} \mathbb{1}_{\Lambda}\right)=\operatorname{tr} h_{\alpha}\left(\mathrm{P}_{\ell}(\Lambda)\right)=\operatorname{tr} \mathbb{1}_{\Lambda} h_{\alpha}\left(\mathrm{P}_{\ell}(\Lambda)\right) \mathbb{1}_{\Lambda}
$$

and call it the local $\alpha$-Rényi ground-state entropy of the $\ell$ th Landau level (with chemical potential $\mu \geq(2 \ell+1) B)$. Obviously, we have $S_{\alpha, 0}(\Lambda)=S_{\alpha}(\Lambda)$ if $0<B \leq \mu<3 B$. Along with Theorem 8 the following result holds

Theorem 10. (Asymptotics of the local Rényi ground-state entropies of the $\ell$ th Landau level) Let $\Lambda \subset \mathbb{R}^{2}$ be a bounded $\mathrm{C}^{3}$-region, $\ell \in \mathbb{N}_{0}$, and $\mu \geq(2 \ell+1) B$. Then the local $\alpha$-Rényi ground-state entropy of the $\ell$ th Landau level (4.6) obeys

$$
S_{\alpha, \ell}(L \Lambda)=L \sqrt{B}|\partial \Lambda| \mathrm{M}_{\ell}\left(h_{\alpha}\right)+o(L),
$$

as $L \rightarrow \infty$. The asymptotic coefficient $\mathrm{M}_{\ell}\left(h_{\alpha}\right)$ is given by (2.10). It is finite and positive.

The remark immediately below Theorem 8 applies analogously to this theorem when (2.14) is replaced with (2.13). The proof of Theorem 10 builds on Theorem 1 and repeats the proof of Theorem 8 in Subsection 4.3 with $\mathrm{P}_{\leq v}$ replaced by $\mathrm{P}_{\ell}$.

The next subsection contains estimates being crucial for the proof of the above results.

4.2. Estimates for singular values. We begin by introducing certain operators $\mathrm{T}_{r, R}$ on $\mathrm{L}^{2}\left(\mathbb{R}^{2}\right)$. To this end, we denote by $\mathrm{D}(x, R) \subset \mathbb{R}^{2}$ the open disk of radius $R>0$, centered at the point $x \in \mathbb{R}^{2}$ and abbreviate $\mathbb{1}_{R}:=\mathbb{1}_{\mathrm{D}(0, R)}$ and similarly with $R$ replaced by $r>0$. Then we define the operators

$$
\mathrm{T}_{r, R}:=\mathrm{T}_{r, R}^{(\ell)}:=\mathbb{1}_{r} \mathrm{P}_{\ell}\left(\mathbb{1}-\mathbb{1}_{R}\right), \quad \mathrm{T}_{r, 0}:=\mathbb{1}_{r} \mathrm{P}_{\ell}, \quad \ell \in \mathbb{N}_{0} .
$$

Here we assume that the magnetic-field strength has been "scaled out", so that $B=1$ in formula (2.2). We interpret $\mathrm{T}_{r, R}$ as an operator from $\mathrm{L}^{2}\left(\mathbb{R}^{2}\right)$ into $\mathrm{L}^{2}(\mathrm{D}(0, r))$.

In order to estimate the singular values of $\mathrm{T}_{r, R}$ we recall the short compilation below Lemma 4 and a classical result due to Birman and Solomyak, see [1, Theorem 4.7]. We quote the required fact in a form adapted to our purposes.

Proposition 11. Let $\mathrm{Z}: \mathrm{L}^{2}\left(\mathbb{R}^{2}\right) \rightarrow \mathrm{L}^{2}(\mathrm{D}(0, r))$ be an integral operator defined by $a$ complex-valued kernel $\mathcal{Z}(x, y)$ obeying

$$
N_{\gamma}(\mathcal{Z}):=\left[\sum_{0 \leq s, t \leq \gamma} \int_{\mathbb{R}^{2}} \mathrm{~d} y \int_{\mathrm{D}(0, r)} \mathrm{d} x\left|\frac{\partial^{s}}{\partial x_{1}^{s}} \frac{\partial^{t}}{\partial x_{2}^{t}} \mathcal{Z}(x, y)\right|^{2}\right]^{\frac{1}{2}}<\infty,
$$

for some $\gamma \in \mathbb{N}_{0}$. Then the singular values $s_{n}(\mathrm{Z})$ of $\mathrm{Z}$ satisfy the bound

$$
s_{n}(\mathrm{Z}) \leq C n^{-\frac{1+\gamma}{2}} N_{\gamma}(\mathcal{Z}), \quad n \in \mathbb{N}
$$

with a positive constant $C$ dependent on $r$ but independent of the kernel $\mathcal{Z}$.

Lemma 12. The operator $\mathrm{T}_{r, 0}$ belongs to the Schatten-von Neumann class $\mathfrak{S}_{p}$ for all $p>0$. Moreover, if $R>r$, then

$$
\left\|\mathrm{T}_{r, R}\right\|_{p} \leq C \exp \left(-(R-r)^{2} / 8\right),
$$

with some constant $C$ dependent on $r$ but independent of $R$. 
Proof. In order to apply Proposition 11, we estimate for $\|x\|<r$, and $s, t \in \mathbb{N}_{0}$, $0 \leq s, t \leq \gamma$ :

$$
\left|\frac{\partial^{s}}{\partial x_{1}^{s}} \frac{\partial^{t}}{\partial x_{2}^{t}} p_{\ell}(x, y)\right| \leq C_{\gamma, \ell}(1+\|y\|)^{\gamma}\left(1+\|x-y\|^{2}\right)^{\ell} \exp \left(-\|x-y\|^{2} / 4\right),
$$

with a constant $C_{\gamma, \ell}$ depending on $r$. Here we have used the fact that $\mathcal{L}_{\ell}$ is a polynomial of degree $\ell$. For $R>r$ and $y \notin \mathrm{D}(0, R)$ we conclude that

$$
\left|\frac{\partial^{s}}{\partial x_{1}^{s}} \frac{\partial^{t}}{\partial x_{2}^{t}} p_{\ell}(x, y)\right| \leq C_{\gamma, \ell}(1+\|y\|)^{\gamma}\left(1+\|x-y\|^{2}\right)^{\ell} \exp \left(-(R-r)^{2} / 8\right) \exp \left(-\|x-y\|^{2} / 8\right) .
$$

Thus the integral kernel $\mathcal{T}_{r, R}(x, y):=1_{r}(x) p_{\ell}(x, y)\left(1-1_{R}(y)\right)$ of $\mathrm{T}_{r, R}$ satisfies, for any $\gamma \in \mathbb{N}$, the bounds

$$
\begin{aligned}
& N_{\gamma}\left(\mathcal{T}_{r, R}\right) \leq C_{\gamma, \ell}, \text { for any } R \geq 0, \\
& N_{\gamma}\left(\mathcal{T}_{r, R}\right) \leq C_{\gamma, \ell} \exp \left(-(R-r)^{2} / 8\right), \text { if } R>r,
\end{aligned}
$$

where the constant $C_{\gamma, \ell}$ is independent of $R$. For an arbitrary $p>0$ we now take $\gamma>2 p^{-1}-1$. Then by Proposition $11, \mathrm{~T}_{r, R} \in \mathfrak{S}_{p}$ for all $R \geq 0$ and the bound (4.9) holds for $R>r$.

Lemma 12 is an important ingredient to bound quasi-norms of the operator $\mathbb{1}_{L \Lambda} \mathrm{P}_{\ell}(\mathbb{1}-$ $\left.\mathbb{1}_{L \Lambda}\right)$. Although our main result Theorem 8 is proved for a bounded $C^{3}$-region $\Lambda$, the next theorem even holds for a bounded Lipschitz region. Here, the boundary curve $\partial \Lambda$ of $\Lambda$ is Lipschitz continuous.

Theorem 13. Let $\Lambda \subset \mathbb{R}^{2}$ be a bounded Lipschitz region and $\ell \in \mathbb{N}_{0}$. Moreover, let $p \in(0,1]$ and $L_{0}>0$ finite. Then there exists a constant $C$, depending only on $\Lambda$ and $L_{0}$, such that for any $L \geq L_{0}$,

$$
\left\|\mathbb{1}_{L \Lambda} \mathrm{P}_{\ell}\left(\mathbb{1}-\mathbb{1}_{L \Lambda}\right)\right\|_{p}^{p} \leq C L .
$$

Proof. We begin with two useful observations. Firstly, we note that $\| \mathbb{1}_{L \Lambda} \mathrm{P}_{\ell}(\mathbb{1}-$ $\left.\mathbb{1}_{L \Lambda}\right)\left\|_{p} \leq\right\| \mathrm{T}_{L r, 0} \|_{p}$ by (2.17) with some $r>0$, where the operator $\mathrm{T}_{r, 0}$ is defined in (4.8). Thus, by Lemma 12, for every fixed $L$ the left-hand side of the claim (4.10) is finite. Consequently, it suffices to prove (4.10) for $L \geq L_{0}$ with an arbitrary choice of the finite $L_{0}$.

Secondly, denoting $\Lambda^{\prime}:=L_{0} \Lambda$, we can rewrite (4.10) as follows:

$$
\left\|\mathbb{1}_{L \Lambda^{\prime}} \mathrm{P}_{\ell}\left(\mathbb{1}-\mathbb{1}_{L \Lambda^{\prime}}\right)\right\|_{p}^{p} \leq C L, \quad L \geq 1,
$$

where $C$ depends on $\Lambda$ and the arbitrary $L_{0}$.

Now we can proceed with the proof. We cover $\Lambda$ by finitely many disks $\mathrm{D}\left(x_{k}, r_{k}\right)$ such that either

(1) $x_{k} \in \partial \Lambda$ and inside each disk $\mathrm{D}\left(x_{k}, 8 r_{k}\right)$, with an appropriate choice of coordinates, the domain $\Lambda$ is given locally by the epigraph of a Lipschitz function (see below), or

(2) $\mathrm{D}\left(x_{k}, r_{k}\right) \subset \Lambda$ and $\operatorname{dist}\left(\mathrm{D}\left(x_{k}, r_{k}\right), \Lambda^{\complement}\right)>0$, where $\Lambda^{\complement}:=\mathbb{R}^{2} \backslash \Lambda$ denotes the complement of $\Lambda$. 
It is clear that we may assume that all radii $r_{k}$ are equal to each other. Moreover, by replacing $\Lambda$ with $\Lambda^{\prime}=L_{0} \Lambda$ with $L_{0}=r_{k}^{-1}$, we may assume that $r_{k}=1$. This equality holds throughout the proof.

Case (1): We fix one disk $\mathrm{D}:=\mathrm{D}\left(x_{k}, 1\right) \subset \mathbb{R}^{2}, x_{k} \in \partial \Lambda$, and denote $\widetilde{\mathrm{D}}:=\mathrm{D}\left(x_{k}, 8\right)$. Let $\Phi: \mathbb{R} \rightarrow \mathbb{R}$ be a Lipschitz function such that

$$
\Lambda \cap \widetilde{\mathrm{D}}=\left\{x=\left(x^{\prime}, x^{\prime \prime}\right) \in \mathbb{R}^{2}: x^{\prime \prime}>\Phi\left(x^{\prime}\right)\right\} \cap \widetilde{\mathrm{D}} .
$$

By $M \geq 0$ we denote the Lipschitz constant for $\Phi$, i.e.

$$
|\Phi(t)-\Phi(u)| \leq M|t-u|, \quad t, u \in \mathbb{R} .
$$

It is clear that

$$
\begin{aligned}
& (L \Lambda \cap L D) \subset(L \Lambda \cap L \widetilde{D})=\left\{x=\left(x^{\prime}, x^{\prime \prime}\right): x^{\prime \prime}>\Phi_{L}\left(x^{\prime}\right)\right\} \cap L \widetilde{D}, \\
& \Phi_{L}(t):=L \Phi\left(t L^{-1}\right), \quad t \in \mathbb{R},
\end{aligned}
$$

and that the Lipschitz constant for $\Phi_{L}$ also equals $M$. Without loss of generality we may assume that $\mathrm{D}=\mathrm{D}(0,1)$ and $\Phi(0)=0$.

Now we construct a covering of $L \Lambda \cap L D$ by open disks. Let $D_{j k}$ be a disk of radius 1 , centered at the point $z_{j, k}:=(j / 2, k / 2) \in L D,(j, k) \in \mathbb{Z}^{2}$. Clearly, such disks form an open covering for $L D$. To extract a convenient covering for $L \Lambda \cap L D$, we define two index sets:

$$
\begin{aligned}
& I_{1}:=\left\{(j, k) \in \mathbb{Z}^{2}: k / 2 \geq \Phi_{L}(j / 2)+2\langle M\rangle, z_{j k} \in L D\right\}, \quad\langle M\rangle:=\sqrt{1+M^{2}}, \\
& I_{2}:=\left\{(j, k) \in \mathbb{Z}^{2}:\left|k / 2-\Phi_{L}(j / 2)\right|<2\langle M\rangle, z_{j k} \in L D\right\} .
\end{aligned}
$$

Since $\Phi$ is Lipschitz, the number of indices in $I_{2}$ obeys $\left|I_{2}\right| \leq C L$ with a constant independent of $L$. The disks $\mathrm{D}_{j k},(j, k) \in I_{1} \cup I_{2}$ form a covering of the intersection $L \Lambda \cap L D$. Observe that for every point $x=\left(x^{\prime}, x^{\prime \prime}\right) \in L \Lambda \cap L D$ we have

$$
\operatorname{dist}\left(x, L \Lambda^{\complement}\right) \leq \min \left\{\left|\Phi_{L}\left(x^{\prime}\right)-x^{\prime \prime}\right|, \operatorname{dist}\left(x, \widetilde{\mathrm{D}}^{\complement}\right)\right\}=\left|\Phi_{L}\left(x^{\prime}\right)-x^{\prime \prime}\right|,
$$

and

$$
\operatorname{dist}\left(x, L \Lambda^{\complement}\right) \geq \min \left\{\langle M\rangle^{-1}\left|x^{\prime \prime}-\Phi_{L}\left(x^{\prime}\right)\right|, \operatorname{dist}\left(x, \widetilde{\mathrm{D}}^{\complement}\right)\right\}=\langle M\rangle^{-1}\left|x^{\prime \prime}-\Phi_{L}\left(x^{\prime}\right)\right|,
$$

so that

$$
R_{j k}:=\operatorname{dist}\left(z_{j k}, L \Lambda^{\complement}\right) \geq\langle M\rangle^{-1}\left(k / 2-\Phi_{L}(j / 2)\right) \geq 2,(j, k) \in I_{1} .
$$

Let $\left(\varphi_{j k}\right)_{j k} \subset \mathrm{C}_{0}^{\infty}\left(\mathbb{R}^{2}\right)$ be a partition of unity subordinate to the constructed covering. In the following, we use a superposed hat for the (bounded) multiplication operator $\widehat{\varphi}$ on $\mathrm{L}^{2}\left(\mathbb{R}^{2}\right)$ uniquely corresponding to $\varphi \in \mathrm{C}_{0}^{\infty}\left(\mathbb{R}^{2}\right)$. We estimate individually the quasi-norms

$$
\left\|\widehat{\varphi}_{j k} \mathbb{1}_{L \Lambda} \mathrm{P}_{\ell}\left(\mathbb{1}-\mathbb{1}_{L \Lambda}\right)\right\|_{p},
$$

for $p \in(0,1]$. Let us consider firstly the set $I_{2}$. For all $(j, k) \in I_{2}$ we have

$$
\left\|\widehat{\varphi}_{j k} \mathbb{1}_{L \Lambda} \mathrm{P}_{\ell}\left(\mathbb{1}-\mathbb{1}_{L \Lambda}\right)\right\|_{p} \leq\left\|\widehat{\varphi}_{j k} \mathbb{1}_{L \Lambda} \mathrm{P}_{\ell}\right\|_{p} \leq\left\|\mathbb{1}_{\mathrm{D}_{j k}} \mathrm{P}_{\ell}\right\|_{p}
$$


The first inequality follows from (2.17). The second inequality holds since $\varphi_{j k} 1_{L \Lambda} \leq$ $1_{\mathrm{D}_{j k}}$ and hence $\widehat{\varphi}_{j k} \mathbb{1}_{L \Lambda} \mathrm{P}_{\ell} \mathbb{1}_{L \Lambda} \widehat{\varphi}_{j k} \leq \mathbb{1}_{\mathrm{D}_{j k}} \mathrm{P}_{\ell} \mathbb{1}_{\mathrm{D}_{j k}}$. Using the standard unitary equivalence of the Hamiltonian (2.1) under "magnetic" translations, we conclude that the right-hand side coincides with $\left\|\mathbb{1}_{D} \mathrm{P}_{\ell}\right\|_{p}$, where $\mathrm{D}=\mathrm{D}(0,1)$, as before. By Lemma 12 , this norm is bounded for all $p>0$, and hence

$$
\left\|\widehat{\varphi}_{j k} \mathbb{1}_{L \Lambda} \mathrm{P}_{\ell}\left(\mathbb{1}-\mathbb{1}_{L \Lambda}\right)\right\|_{p} \leq C
$$

uniformly in $j$ and $k$. Applying the $p$-triangle inequality (2.16) for quasi-norms, we get

$$
\sum_{(j, k) \in I_{2}}\left\|\widehat{\varphi}_{j k} \mathbb{1}_{L \Lambda} \mathrm{P}_{\ell}\left(\mathbb{1}-\mathbb{1}_{L \Lambda}\right)\right\|_{p}^{p} \leq C\left|I_{2}\right| \leq C L .
$$

Suppose now that $(j, k) \in I_{1}$. Thus

$$
\left\|\widehat{\varphi}_{j k} \mathrm{P}_{\ell}\left(\mathbb{1}-\mathbb{1}_{L \Lambda}\right)\right\|_{p} \leq\left\|\mathbb{1}_{\mathrm{D}_{j k}} \mathrm{P}_{\ell}\left(\mathbb{1}-\mathbb{1}_{\mathrm{D}\left(z_{j k}, R_{j k}\right)}\right)\right\|_{p}
$$

with $R_{j k}=\operatorname{dist}\left(z_{j k}, L \Lambda^{\complement}\right) \geq 2$. By the translation argument, the right-hand side coincides with $\left\|\mathrm{T}_{1, R_{j k}}\right\|_{p}$, where the operator $\mathrm{T}_{1, R}$ is defined in (4.8). Consequently, by (4.9),

$$
\left\|\widehat{\varphi}_{j k} \mathrm{P}_{\ell}\left(\mathbb{1}-\mathbb{1}_{L \Lambda}\right)\right\|_{p} \leq C \exp \left(-\left(R_{j k}-1\right)^{2} / 8\right) .
$$

Using the $p$-triangle inequality again, we obtain that

$$
\sum_{(j, k) \in I_{1}}\left\|\widehat{\varphi}_{j k} \mathrm{P}_{\ell}\left(\mathbb{1}-\mathbb{1}_{L \Lambda}\right)\right\|_{p}^{p} \leq C \sum_{(j, k) \in I_{1}} \exp \left(-p\left(R_{j k}-1\right)^{2} / 8\right) .
$$

Employing (4.11), for any fixed $j$, the summation over $k$ yields the estimate

$$
\sum_{(j, k) \in I_{1}, j \text { fixed }} \exp \left(-p\left(R_{j k}-1\right)^{2} / 8\right) \leq \sum_{k \in \mathbb{Z}} \mathrm{e}^{-c k^{2}}=C .
$$

Since $|j| \leq 2 L$, the right-hand side of (4.12) does not exceed $C L$. Putting these estimates together we obtain

$$
\left\|\mathbb{1}_{L \mathrm{D}} \mathbb{1}_{L \Lambda} \mathrm{P}_{\ell}\left(\mathbb{1}-\mathbb{1}_{L \Lambda}\right)\right\|_{p}^{p} \leq \sum_{(j, k) \in I_{1} \cup I_{2}}\left\|\widehat{\varphi}_{j k} \mathrm{P}_{\ell}\left(\mathbb{1}-\mathbb{1}_{L \Lambda}\right)\right\|_{p}^{p} \leq C L .
$$

Case (2): We fix one disk $\mathrm{D}=\mathrm{D}\left(x_{k}, r_{k}\right)$ such that $\operatorname{dist}\left(\mathrm{D}, \Lambda^{\mathrm{C}}\right) \geq c$, so that $\operatorname{dist}\left(L D, L \Lambda^{\complement}\right) \geq c L$. We cover $L D$ by unit disks $D_{j}, j=1, \ldots, N$ with $N \leq C L^{2}$ and $\operatorname{dist}\left(\mathrm{D}_{j}, L \Lambda^{\mathrm{C}}\right) \geq c L$. As in the proof of Case (1), we introduce a smooth partition of unity $\left(\varphi_{j}\right)_{j} \subset \mathrm{C}_{0}^{\infty}\left(\mathbb{R}^{2}\right)$ subordinate to this covering, and estimate:

$$
\left\|\widehat{\varphi}_{j} \mathrm{P}_{\ell}\left(\mathbb{1}-\mathbb{1}_{L \Lambda}\right)\right\|_{p}^{p} \leq C \mathrm{e}^{-c L^{2}} .
$$

Consequently, by the $p$-triangle inequality (2.16)

$$
\begin{aligned}
\left\|\mathbb{1}_{L D} \mathrm{P}_{\ell}\left(\mathbb{1}-\mathbb{1}_{L \Lambda}\right)\right\|_{p}^{p} & \leq \sum_{j}\left\|\widehat{\varphi}_{j} \mathrm{P}_{\ell}\left(\mathbb{1}-\mathbb{1}_{L \Lambda}\right)\right\|_{p}^{p} \\
& \leq C \sum_{j} \mathrm{e}^{-c L^{2}} \leq C L^{2} \mathrm{e}^{-c L^{2}} \leq C \mathrm{e}^{-c^{\prime} L^{2}} .
\end{aligned}
$$

To complete the proof we add the estimates of the form (4.13) and (4.14) for all disks covering $\Lambda$, using the $p$-triangle inequality again. 
4.3. Proofs of Lemma 7 and Theorem 8. We use the bound

$$
0 \leq h_{\alpha}(t) \leq C_{\alpha} t^{\beta}(1-t)^{\beta}, \quad t \in[0,1],
$$

with a positive constant $C_{\alpha}<\infty$. Here we choose $\beta=\alpha$ if $\alpha<1$, any $\beta \in(0,1)$ if $\alpha=1$, and $\beta=1$ if $\alpha>1$. Since $\Lambda$ is bounded, we have $\Lambda \subset \mathrm{D}(0, r)$ with some $r>0$. This leads to the estimate

$$
S_{\alpha}(\Lambda) \leq C_{\alpha} \operatorname{tr}\left[\mathrm{P}_{\leq v}(\Lambda)^{\beta}\left(\mathbb{1}-\mathrm{P}_{\leq v}(\Lambda)\right)^{\beta}\right] \leq C_{\alpha}\left\|\mathbb{1}_{\Lambda} \mathrm{P}_{\leq v}\left(\mathbb{1}-\mathbb{1}_{\Lambda}\right)\right\|_{\beta}^{\beta} \leq C_{\alpha}\left\|\mathbb{1}_{r} \mathrm{P}_{\leq v}\right\|_{\beta}^{\beta} .
$$

Using the $\beta$-triangle inequality and Lemma 12 , we obtain the estimate (see (4.8) for the definition of $\mathrm{T}_{r, 0}^{(\ell)}$ )

$$
S_{\alpha}(\Lambda) \leq C_{\alpha} \sum_{\ell=0}^{v}\left\|\mathrm{~T}_{r, 0}^{(\ell)}\right\|_{\beta}^{\beta}<\infty,
$$

as claimed. This proves Lemma 7.

Now we prove Theorem 8. Since $h_{\alpha}$ satisfies the bound (4.15), it follows from Lemma 4 that the coefficient $\mathrm{M}_{\leq v}\left(h_{\alpha}\right)$ is finite. The positivity of $\mathbf{M}_{\leq v}\left(h_{\alpha}\right)$ is obvious from $h_{\alpha} \geq 0, \mathcal{K}_{v, \xi} \geq 0$, and $h_{\alpha}(1)=0$. If $\alpha>1$, then the function $h_{\alpha}$ is $C^{1}$-smooth on $[0,1]$, and hence the claim follows immediately from Theorem 2 .

In order to prove (4.3) for $\alpha \leq 1$ we follow the method of [16]. For $\varepsilon>0$ we choose a smooth function $\zeta_{\varepsilon}$ such that $0 \leq \zeta_{\varepsilon} \leq 1$ and

$$
\zeta_{\varepsilon}(t)= \begin{cases}1 & \text { if } t \in[0, \varepsilon / 2] \cup[1-\varepsilon / 2,1], \\ 0 & \text { if } t \in[\varepsilon, 1-\varepsilon] .\end{cases}
$$

In view of estimate (4.15), we have

$$
\left|\left(\zeta_{\varepsilon} h_{\alpha}\right)(t)\right| \leq C \varepsilon^{r}[t(1-t)]^{r}, \quad r:=\frac{\beta}{2},
$$

and hence

$$
\left\|\left(\zeta_{\varepsilon} h_{\alpha}\right)\left(\mathrm{P}_{\leq \nu}(L \Lambda)\right)\right\|_{1} \leq C \varepsilon^{r}\left\|\mathrm{P}_{\leq \nu}(L \Lambda)\left(\mathbb{1}-\mathrm{P}_{\leq \nu}(L \Lambda)\right)\right\|_{r}^{r}=C \varepsilon^{r}\left\|\mathbb{1}_{L \Lambda} \mathrm{P}_{\leq v}\left(\mathbb{1}-\mathbb{1}_{L \Lambda}\right)\right\|_{2 r}^{2 r}
$$

By Theorem 13, the quasi-norm on the right-hand side does not exceed CL. Consequently,

$$
\left|\operatorname{tr}\left[\left(\zeta_{\varepsilon} h_{\alpha}\right)\left(\mathrm{P}_{\leq v}(L \Lambda)\right)\right]\right| \leq C \varepsilon^{r} L .
$$

On the other hand, the function $\left(1-\zeta_{\varepsilon}\right) h_{\alpha}$ vanishes in a vicinity of 0 and 1 and, therefore, by Theorem 2, we have

$$
\operatorname{tr}\left[\left(1-\zeta_{\varepsilon}\right) h_{\alpha}\left(\mathrm{P}_{\leq v}(L \Lambda)\right)\right]=L \sqrt{B}|\partial \Lambda| \mathrm{M}_{\leq \nu}\left(\left(1-\zeta_{\varepsilon}\right) h_{\alpha}\right)+o(L), \quad L \rightarrow \infty .
$$

By (4.16),

$$
\mathrm{M}_{\leq \nu}\left(h_{\alpha}\right)-\mathrm{M}_{\leq v}\left(\left(1-\zeta_{\varepsilon}\right) h_{\alpha}\right)=\mathrm{M}_{\leq v}\left(\zeta_{\varepsilon} h_{\alpha}\right) \leq C \varepsilon^{r} \mathrm{M}_{\leq v}\left(\tilde{g}^{r}\right), \quad \tilde{g}(t)=t(1-t) .
$$

Combining (4.18), (4.19), and (4.20) gives

$$
\limsup _{L \rightarrow \infty}\left|\frac{\operatorname{tr} h_{\alpha}\left(\mathrm{P}_{\leq \nu}(L \Lambda)\right)}{L}-\sqrt{B}\right| \partial \Lambda\left|\mathrm{M}_{\leq \nu}\left(h_{\alpha}\right)\right| \leq C \varepsilon^{r} .
$$

Since $\varepsilon>0$ is arbitrary, this yields the claim. 


\section{On an Improvement to Sub-leading Terms}

Without going into details, we show here how one can improve the asymptotic expansions in Lemmas 5 and 6 to

$$
\begin{aligned}
& \operatorname{tr} f\left(\mathrm{P}_{\ell}(L \Lambda)\right)=L^{2} B \frac{|\Lambda|}{2 \pi} f(1)+L \sqrt{B}|\partial \Lambda| \mathrm{M}_{\ell}(f)+o(1), \\
& \operatorname{tr} f\left(\mathrm{P}_{\leq n}(L \Lambda)\right)=L^{2} B \frac{|\Lambda|}{2 \pi}(n+1) f(1)+L \sqrt{B}|\partial \Lambda| \mathrm{M}_{\leq n}(f)+o(1),
\end{aligned}
$$

where $f(t)=t^{n}, n=1,2, \ldots$ This follows again by proving the corresponding statements for all natural powers of $\mathrm{P}_{\ell}(L \Lambda)$. Here, we use the expansion of Roccaforte in Proposition 14 up to the second order $\varepsilon^{2}$. For any vector $y=-z \mathrm{~J} n_{x}+t n_{x} \in \mathbb{R}^{2}$, written in the form (3.8), we have $\|y\|^{2}-2\left\langle y \mid n_{x}\right\rangle^{2}=z^{2}-t^{2}$. Then the $W$-term in (3.7) takes the form

$$
\begin{aligned}
\sum_{q=1}^{m-1} \int_{\mathcal{S}_{q}} \mathrm{dA}(x) \kappa(x) & \int_{\mathbb{R}^{m-1}} \mathrm{~d} \mathbf{z} \int_{\mathbb{R}^{m-1}} \mathrm{~d} \mathbf{t}\left[\left(\sum_{j=1}^{q} z_{j}\right)^{2}-\left(\sum_{j=1}^{q} t_{j}\right)^{2}\right] \exp \left(\mathrm{i} \frac{B}{2}\langle\mathbf{z} \mid \mathrm{S} \mathbf{t}\rangle\right) \\
& \times \prod_{j=1}^{m} \mathcal{L}_{\ell}\left(\left(z_{j}^{2}+t_{j}^{2}\right) / 2\right) g\left(z_{j}\right) g\left(t_{j}\right)+o(1) .
\end{aligned}
$$

If we exchange the $\mathbf{z}$ and $\mathbf{t}$ variables, the integrand is seen to be almost anti-symmetric except for the sign in the exponent. This can be remedied by changing, for instance, $t$ to -t. Hence, the integral in (5.3) vanishes by symmetry.

At present we do not know how to extend the expansions (5.1) and (5.2) to the entropy function $f=h_{\alpha}$, but believe that the corresponding term vanishes also in this case. In other words, there is zero topological (entanglement) entropy, see [12,18].

Acknowledgements Various parts of this paper were developed and written during several visits of HL and AVS to the FernUniversität in Hagen in 2016-2019, and during the stay of all authors at the International Newton Institute (Cambridge, UK) in 2015 and to the American Institute of Mathematics (AIM) at San Jose (CA, USA) in 2017. The authors are grateful to these institutions for hospitality and financial support. AVS was also supported by the EPSRC grants EP/J016829/1 and EP/P024793/1. We thank Paul Pfeiffer for helpful comments.

Funding Open Access funding enabled and organized by Projekt DEAL.

Open Access This article is licensed under a Creative Commons Attribution 4.0 International License, which permits use, sharing, adaptation, distribution and reproduction in any medium or format, as long as you give appropriate credit to the original author(s) and the source, provide a link to the Creative Commons licence, and indicate if changes were made. The images or other third party material in this article are included in the article's Creative Commons licence, unless indicated otherwise in a credit line to the material. If material is not included in the article's Creative Commons licence and your intended use is not permitted by statutory regulation or exceeds the permitted use, you will need to obtain permission directly from the copyright holder. To view a copy of this licence, visit http://creativecommons.org/licenses/by/4.0/.

Publisher's Note Springer Nature remains neutral with regard to jurisdictional claims in published maps and institutional affiliations.

\section{A. Roccaforte's Formula for the Area of Intersections}

We recall Roccaforte's results in [25, Corollary 2.4, Lemma 2.5] for the special case of the Euclidean plane. Since the boundary curve $\partial \Lambda$ is a one-dimensional manifold, the 
second fundamental form of $\partial \Lambda$ is just its curvature. Therefore, his formula takes the simpler form given in Proposition 14 below.

In our application we scale out $\varepsilon$ and identify $L \sqrt{B}=1 / \varepsilon$. For given points/vectors $v_{1}, \ldots, v_{r}$ in $\mathbb{R}^{2}$ we denote by $\Lambda_{\varepsilon}:=\Lambda \cap\left(\Lambda+\varepsilon v_{1}\right) \cap \cdots \cap\left(\Lambda+\varepsilon v_{r}\right)$ the intersection of $\Lambda$ with its $r$ translates.

Proposition 14. Let $\Lambda \subset \mathbb{R}^{2}$ be a bounded $\mathrm{C}^{3}$-region. Then for arbitrary $\left(v_{1}, \ldots, v_{r}\right) \in$ $\mathbb{R}^{2} \times \cdots \times \mathbb{R}^{2}$, we have

$$
|| \Lambda \backslash \Lambda_{\varepsilon}\left|-\varepsilon \int_{\partial \Lambda} \mathrm{d} A(x) \max \left\{0,\left\langle v_{1} \mid n_{x}\right\rangle, \ldots,\left\langle v_{r} \mid n_{x}\right\rangle\right\}\right| \leq C \varepsilon^{2} \sum_{j=1}^{r}\left\|v_{j}\right\|^{2}
$$

with some constant $C$ independent of $\varepsilon>0$ or $v_{1}, v_{2}, \ldots, v_{r}$, where $n_{x}$ is the inward unit normal to the curve $\partial \Lambda$. Moreover, for all $\left(v_{1}, \ldots, v_{r}\right) \in \mathbb{R}^{2} \times \cdots \times \mathbb{R}^{2}$ except for a set of $2 r$-dimensional Lebesgue measure zero we have

$$
\begin{aligned}
\left|\Lambda \backslash \Lambda_{\varepsilon}\right|= & \varepsilon \int_{\partial \Lambda} \mathrm{d} A(x) \max \left\{0,\left\langle v_{1} \mid n_{x}\right\rangle, \ldots,\left\langle v_{r} \mid n_{x}\right\rangle\right\} \\
& +\frac{1}{2} \varepsilon^{2} \sum_{q=1}^{r} \int_{\mathcal{C}_{q}} \mathrm{~d} A(x) \kappa(x)\left[\left\|v_{q}\right\|^{2}-2\left\langle v_{q} \mid n_{x}\right\rangle^{2}\right]+o\left(\varepsilon^{2}\right), \varepsilon \rightarrow 0 .
\end{aligned}
$$

Here, $\mathcal{C}_{q}:=\left\{x \in \partial \Lambda:\left\langle v_{q} \mid n_{x}\right\rangle=\max \left\{0,\left\langle v_{1} \mid n_{x}\right\rangle, \ldots,\left\langle v_{r} \mid n_{x}\right\rangle\right\}\right\}$ and $\kappa(x)$ is the curvature of $\partial \Lambda$ at the point $x \in \partial \Lambda$.

\section{B. Miscellaneous Identities}

B.1. Proof of the result (3.26) of a change of variables. The term quadratic in $\xi$ is obviously correct and we start with the linear term in $\xi$. The inverse of the matrix $\mathrm{A}:=\mathrm{A}^{(q)}$ has the entries

$$
\mathrm{A}^{-1}(i, j)=\left\{\begin{aligned}
1 & \text { if } i=j \\
-1 & \text { if } 1 \leq i=j-1 \leq q-1 \\
-1 & \text { if } q+1 \leq j=i-1 \leq m-2 \\
0 & \text { otherwise }
\end{aligned}\right.
$$


and

$$
\begin{aligned}
\sum_{i=1}^{m-1} T_{i} & =\sum_{j=1}^{m-1} t_{j} \sum_{i=1}^{m-1} \mathrm{~S}_{i j} \\
& =\sum_{j=1}^{m-1}\left(\mathrm{~A}^{-1} \boldsymbol{\tau}\right)_{j}(m-2 j) \\
& =\sum_{j=1}^{m-1}(m-2 j) \tau_{j}-\sum_{j=1}^{q-1}(m-2 j) \tau_{j+1}-\sum_{j=q+2}^{m-1}(m-2 j) \tau_{j-1} \\
& =(m-2) \tau_{1}-2 \sum_{j=2}^{q} \tau_{j}+2 \sum_{j=q+1}^{m-2} \tau_{j}-(m-2) \tau_{m-1} \\
& \rightsquigarrow(m-2) \tau_{1}-2 \sum_{j=2}^{q} \tau_{j}-2 \sum_{j=q+1}^{m-2} \tau_{j}+(m-2) \tau_{m-1} \\
& =(m-2)\left(\tau_{1}+\tau_{m-1}\right)-2 \sum_{j=2}^{m-2} \tau_{j} .
\end{aligned}
$$

In the $\rightsquigarrow$ line we have reversed the signs of $\tau_{q+1}, \ldots, \tau_{m-1}$. Now we replace $\xi$ on the left-hand side of (3.26) by $\xi-\left(\tau_{1}+\tau_{m-1}\right) / 2$, which finally yields the linear term

$$
-m \xi\left(\tau_{1}+\tau_{m-1}\right)+\xi\left[(m-2)\left(\tau_{1}+\tau_{m-1}\right)-2 \sum_{j=2}^{m-2} \tau_{j}\right]=-2 \xi \sum_{j=1}^{m-1} \tau_{j} .
$$

Similarly, since

$$
\sum_{1 \leq j \leq m-1} \mathrm{~S}_{j k} \mathrm{~S}_{j \ell}= \begin{cases}m-2 & \text { if } k=\ell \\ m-1-2|k-\ell| & \text { if } k \neq \ell\end{cases}
$$

we have $\left(T_{m}=0\right)$

$$
\begin{aligned}
\sum_{1 \leq j \leq m-1} T_{j}^{2} & +\sum_{1 \leq j \leq m} t_{j}^{2}=\sum_{1 \leq k, \ell \leq m-1} t_{k} t_{\ell} \sum_{1 \leq j \leq m-1} \mathrm{~S}_{j k} \mathrm{~S}_{j \ell}+\sum_{1 \leq k \leq m-1} t_{k}^{2}+t_{m}^{2} \\
& =(m-1) \sum_{1 \leq k \leq m-1} t_{k}^{2}+\left(\sum_{1 \leq k \leq m-1} t_{k}\right)^{2}+2 \sum_{1 \leq k<\ell \leq m-1} t_{k} t_{\ell}(m-1-2(\ell-k)) \\
& =m\left(\sum_{1 \leq k \leq m-1} t_{k}\right)^{2}-4 \sum_{1 \leq k<\ell \leq m-1} t_{k} t_{\ell}(\ell-k) \\
& =m\left(\tau_{1}+\tau_{m-1}\right)^{2}-4 \sum_{1 \leq k<\ell \leq m-1}\left(\mathrm{~A}^{-1} \boldsymbol{\tau}\right)(k)\left(\mathrm{A}^{-1} \boldsymbol{\tau}\right)(\ell)(\ell-k)
\end{aligned}
$$


Next, we write

$$
\begin{aligned}
\sum_{1 \leq k<\ell \leq m-1}\left(\mathrm{~A}^{-1} \tau\right)(k)\left(\mathrm{A}^{-1} \boldsymbol{\tau}\right)(\ell)(\ell-k) & =\sum_{1 \leq k<\ell \leq q-1}\left(\tau_{k}-\tau_{k+1}\right)\left(\tau_{\ell}-\tau_{\ell+1}\right)(\ell-k) \\
& +\sum_{1 \leq k \leq q-1}\left(\tau_{k}-\tau_{k+1}\right) \tau_{q}(q-k) \\
& +\sum_{1 \leq k \leq q-1}\left(\tau_{k}-\tau_{k+1}\right) \tau_{q+1}(q+1-k)+\tau_{q} \tau_{q+1} \\
& +\sum_{1 \leq k \leq q-1, q+2 \leq \ell}\left(\tau_{k}-\tau_{k+1}\right)\left(\tau_{\ell}-\tau_{\ell-1}\right)(\ell-k) \\
& +\sum_{q+2 \leq k<\ell \leq m-1}\left(\tau_{k}-\tau_{k-1}\right)\left(\tau_{\ell}-\tau_{\ell-1}\right)(\ell-k) \\
& +\tau_{q} \tau_{q+1}+\tau_{q} \sum_{q+2 \leq \ell \leq m-1}\left(\tau_{\ell}-\tau_{\ell-1}\right)(\ell-q) \\
& +\tau_{q+1} \sum_{q+2 \leq \ell \leq m-1}\left(\tau_{\ell}-\tau_{\ell-1}\right)(\ell-q-1) .
\end{aligned}
$$

Now we reverse the signs of $\tau_{q+1}, \ldots, \tau_{m-1}$. To this end, let $\mathrm{I}:=\mathrm{I}^{(q)}=\operatorname{diag}(\underbrace{1, \ldots, 1}_{q}$, $\underbrace{-1, \ldots,-1}_{m-1-q})$ be the diagonal $(m-1) \times(m-1)$ matrix that provides this reversal. Then we get

$$
\begin{aligned}
\sum_{1 \leq k<\ell \leq m-1}\left(\mid \mathrm{A}^{-1} \boldsymbol{\tau}\right)(k)\left(\mid \mathrm{A}^{-1} \boldsymbol{\tau}\right)(\ell)(\ell-k) & =\sum_{1 \leq k<\ell \leq q-1}\left(\tau_{k}-\tau_{k+1}\right)\left(\tau_{\ell}-\tau_{\ell+1}\right)(\ell-k) \\
& +\tau_{q}\left(\tau_{1}(q-1)-\sum_{2 \leq k \leq q} \tau_{k}\right) \\
& -\tau_{q+1}\left(\tau_{1} q-\sum_{2 \leq k \leq q} \tau_{k}\right) \\
& -\sum_{1 \leq k \leq q-1, q+2 \leq \ell}\left(\tau_{k}-\tau_{k+1}\right)\left(\tau_{\ell}-\tau_{\ell-1}\right)(\ell-k) \\
& +\sum_{q+2 \leq k<\ell \leq m-1}\left(\tau_{k}-\tau_{k-1}\right)\left(\tau_{\ell}-\tau_{\ell-1}\right)(\ell-k) \\
& -\tau_{q} \tau_{q+1}-\tau_{q} \sum_{q+2 \leq \ell \leq m-1}\left(\tau_{\ell}-\tau_{\ell-1}\right)(\ell-q) \\
& +\tau_{q+1} \sum_{q+2 \leq \ell \leq m-1}\left(\tau_{\ell}-\tau_{\ell-1}\right)(\ell-q-1) .
\end{aligned}
$$

Recall that $\xi$ in (3.26) is replaced by $\xi-\left(\tau_{1}+\tau_{m-1}\right) / 2$. That is, on top of the term $\frac{1}{4} \sum_{1 \leq j \leq m}\left(T_{j}^{2}+t_{j}^{2}\right.$ ) (after reversing signs of $\left.\tau_{q+1}, \ldots, \tau_{m-1}\right)$ we also have the term

$$
\begin{gathered}
m\left(\frac{1}{2}\left(\tau_{1}+\tau_{m-1}\right)\right)^{2}-\frac{1}{2}\left(\tau_{1}+\tau_{m-1}\right)\left[(m-2)\left(\tau_{1}+\tau_{m-1}\right)-2 \sum_{2 \leq j \leq m-2} \tau_{j}\right] \\
=\left(\tau_{1}+\tau_{m-1}\right)^{2}\left(1-\frac{m}{4}\right)+\left(\tau_{1}+\tau_{m-1}\right) \sum_{2 \leq j \leq m-2} \tau_{j} .
\end{gathered}
$$


Let $\mathrm{B}$ be the $(m-1) \times(m-1)$ matrix defined as

$$
\begin{aligned}
\langle\boldsymbol{\tau}, \mathrm{B} \boldsymbol{\tau}\rangle: & =\frac{m}{4}\left(\tau_{1}-\tau_{m-1}\right)^{2}-\sum_{1 \leq k<\ell \leq m-1}\left(\mathrm{IA}^{-1} \boldsymbol{\tau}\right)(k)\left(\mathrm{IA}^{-1} \boldsymbol{\tau}\right)(\ell)(\ell-k) \\
& +\left(\tau_{1}+\tau_{m-1}\right)^{2}\left(1-\frac{m}{4}\right)+\left(\tau_{1}+\tau_{m-1}\right) \sum_{2 \leq j \leq m-2} \tau_{j}
\end{aligned}
$$

Then we need to show that $\mathrm{B}=\nVdash$. We distinguish between certain ranges of indices.

- $i_{0}=j_{0}=1$ : From the first and last term in the definition of $\mathrm{B}$ we get $\mathrm{B}_{1,1}=$ $\frac{m}{4}+\left(1-\frac{m}{4}\right)=1 \mathfrak{d}$

- $1=i_{0}<j_{0} \leq q-1$ : choose in (B.2) $k=1, \ell=j_{0}$ or $k=1, \ell=j_{0}-1$. Then we have $\mathrm{B}_{1, j_{0}}=1-\left(j_{0}-i_{0}\right)+\left(j_{0}-1-i_{0}\right)=0 \checkmark$

- $i_{0}=1, j_{0}=q$ : choose in (B.2) $k=1, \ell=q-1$. Then, together with (B.3) and the last term in the definition of $\mathrm{B}$ we get $\mathrm{B}_{1, q}=q-2-(q-1)+1=0 \checkmark$

- $i_{0}=1, j_{0}=q+1$ : choose in (B.5) $k=1, \ell=q+2$. Then, together with (B.4) and the last term in the definition of $\mathrm{B}$ we get $\mathrm{B}_{1, q+1}=q-(q+1)-1=0 \checkmark$

- $i_{0}=1, q+2 \leq j_{0} \leq m-1$ : choose in (B.5) $k=1, \ell=j_{0}$ and $k=1, \ell=j_{0}+1$. Then, together with the last term in the definition of $\mathrm{B}$ we get $\mathrm{B}_{1, j_{0}}=j_{0}-1-j_{0}+1=$ $0 \checkmark$

- $2 \leq i_{0}=j_{0} \leq q-1$ : choose in (B.2) $k=i_{0}-1, \ell=i_{0}$. Then $\mathrm{B}_{i_{0}, i_{0}}=1 \boldsymbol{\checkmark}$

- $2 \leq i_{0}<j_{0} \leq q-1$ : choose in (B.2) $k=i_{0}, \ell=j_{0}, k=i_{0}-1, \ell=j_{0}-1$, $k=i_{0}, \ell=j_{0}-1$, or $k=i_{0}-1, \ell=j_{0}$. Then $\mathrm{B}_{i_{0}, j_{0}}=-\left(j_{0}-i_{0}\right)-\left(j_{0}-i_{0}\right)+$ $\left(j_{0}-1-i_{0}\right)+\left(j_{0}-i_{0}+1\right)=0 \checkmark$

- $2 \leq i_{0} \leq q-1, j_{0}=q$ : choose in (B.2) $\ell=q-1$ and $k=i_{0}$ or $k=i_{0}-1$ and in (B.3) $k=i_{0}$. Then $\mathrm{B}_{i_{0}, q}=q-1-i_{0}-\left(q-1-i_{0}+1\right)+1=0 \checkmark$

- $2 \leq i_{0} \leq q-1, j_{0}=q+1$ : choose in (B.4) $k=i_{0}$ and in (B.5) $\ell=q+2$ and $k=i_{0}$ or $k=i_{0}-1$. Then $\mathrm{B}_{i_{0}, q+1}=-1-\left(q+2-i_{0}\right)+q+2-i_{0}+1=0 \checkmark$

- $2 \leq i_{0} \leq q-1, q+2 \leq j_{0} \leq m-1$ : choose in (B.5) $k=i_{0}$ and $\ell=j_{0}$ or $\ell=j_{0}+1$, or $k=i_{0}-1$ and $\ell=j_{0}$ or $\ell=j_{0}+1$. Then $\mathrm{B}_{i_{0}, j_{0}}=\left(j_{0}-i_{0}\right)-\left(j_{0}+\right.$ $\left.1-i_{0}\right)-\left(j_{0}-i_{0}+1\right)+\left(j_{0}+1-i_{0}+1\right)=0 \checkmark$

- $i_{0}=q, j_{0}=q$ : this term appears in (B.3) if $k=q$. Then $\mathrm{B}_{q, q}=1 \checkmark$

- $i_{0}=q, j_{0}=q+1$ : choose in (B.4) $k=q$, in (B.5) $k=q-1$ and $\ell=q+2$, in (B.7) $\ell=q+2$. Then $\mathrm{B}_{q, q+1}=-1+(q+2-q+1)+1-(q+2-q) 0 \checkmark$

- $i_{0}=q, q+2 \leq j_{0} \leq m-1$ : choose in (B.5) $k=q-1$ and $\ell=j_{0}$ or $\ell=j_{0}+1$ and in (B.7) $\ell=j_{0}$ or $\ell=j_{0}+1$. Then $\mathrm{B}_{q, j_{0}}=-\left(j_{0}-q+1\right)+\left(j_{0}+1-q+1\right)+$ $\left(j_{0}-q\right)-\left(j_{0}+1-q\right)=0 \checkmark$

- $i_{0}=j_{0}=q+1$ : choose in (B.8) $\ell=q+2$. Then $\mathrm{B}_{q+1, q+1}=1 \boldsymbol{J}$

- $i_{0}=q+1, j_{0}=q+2$ : choose in (B.6) $k=q+2, \ell=q+3$ and in (B.8) $\ell=q+2$ or $\ell=q+3$. Then $\mathrm{B}_{q+1, q+2}=-1-1+2=0 \checkmark$

- $i_{0}=q+1, q+2 \leq j_{0} \leq m-1$ : choose in (B.6) $k=q+1$ and $\ell=j_{0}$ or $\ell=j_{0}+1$, and in (B.8) $\ell=j_{0}$ or $\ell=j_{0}+1$. Then $\mathrm{B}_{q+1, j_{0}}=\left(j_{0}-q-1\right)-\left(j_{0}+1-q-1\right)-$ $\left(j_{0}-q-1\right)+\left(j_{0}+1-q-1\right)=0 \checkmark$

- $q+2 \leq i_{0}=j_{0} \leq m-2$ : choose in (B.6) $k=i_{0}$ and $\ell=i_{0}+1$. Then $\mathrm{B}_{i_{0}, i_{0}}=1 \checkmark$

- $i_{0}=j_{0}=m-1: \mathrm{B}_{m-1, m-1}$ comes from the first and third one in the definition of B. That is, $\mathrm{B}_{m-1, m-1}=\frac{m}{4}+\left(1-\frac{m}{4}\right)=1 \checkmark$

- $q+2 \leq i_{0}<j_{0} \leq m-2$ : choose in (B.6) $k=i_{0}, \ell=j_{0}, k=i_{0}+1, \ell=j_{0}$, $k=i_{0}, \ell=j_{0}+1, k=i_{0}+1, \ell=j_{0}+1$. Then $\mathrm{B}_{i_{0}, j_{0}}=-\left(j_{0}-i_{0}\right)+\left(j_{0}-i_{0}-\right.$ $1)+\left(j_{0}+1-i_{0}\right)-\left(j_{0}-i_{0}\right)=0 \checkmark$ 
- $q+2 \leq i_{0}, j_{0}=m-1$ : choose (B.6) $\ell=m-1$ and $k=i_{0}$ or $k=i_{0}+1$. In conjunction with the last term in the definition we obtain $\mathrm{B}_{i_{0}, m-1}=-\left(m-1-i_{0}\right)+$ $\left(m-1-i_{0}-1\right)+1=0 \checkmark$

Finally, switching $\xi$ to $-\xi$ proves (3.26).

B.2. Change of variables in Laguerre polynomials. With $T_{j}$ from (3.13) and $\boldsymbol{\tau}=$ At, $\mathrm{A}=\mathrm{A}^{(q)}$ from (3.25), we claim that

$$
T_{j}=\left\{\begin{array}{ll}
\tau_{1}-\tau_{j}-\tau_{j+1}-\tau_{m-1} & \text { if } 1 \leq j \leq q-1 \\
\tau_{1}-\tau_{q}-\tau_{m-1} & \text { if } j=q \\
\tau_{1}+\tau_{q+1}-\tau_{m-1} & \text { if } j=q+1 \\
\tau_{1}+\tau_{j-1}+\tau_{j}-\tau_{m-1} & \text { if } q+2 \leq j \leq m-1
\end{array} .\right.
$$

To this end, we write

$$
T_{j}=\sum_{1 \leq k \leq j-1}\left(\mathrm{~A}^{-1} \boldsymbol{\tau}\right)(k)-\sum_{j+1 \leq k \leq m-1}\left(\mathrm{~A}^{-1} \boldsymbol{\tau}\right)(k)
$$

and assume for example that $1 \leq j \leq q-1$. Then, using (B.1)

$$
\begin{aligned}
T_{j} & =\sum_{1 \leq k \leq j-1}\left(\tau_{k}-\tau_{k+1}\right)-\sum_{j+1 \leq k \leq q+1}\left(\mathrm{~A}^{-1} \boldsymbol{\tau}\right)(k)-\sum_{j+1 \leq k \leq q+1}\left(\mathrm{~A}^{-1} \boldsymbol{\tau}\right)(k) \\
& =\tau_{1}-\tau_{j}-\sum_{j+1 \leq k \leq q-1}\left(\tau_{k}-\tau_{k+1}\right)-\tau_{q}-\tau_{q+1}-\left(\tau_{m-1}-\tau_{q+1}\right) \\
& =\tau_{1}-\tau_{j}-\tau_{j+1}-\tau_{m-1} .
\end{aligned}
$$

Also,

$$
\begin{aligned}
T_{q} & =\sum_{1 \leq k \leq q-1}\left(\tau_{k}-\tau_{k+1}\right)-\tau_{q+1}-\sum_{q+1 \leq k \leq m-1}\left(\tau_{k}-\tau_{k-1}\right) \\
& =\tau_{1}-\tau_{q}-\tau_{m-1}
\end{aligned}
$$

The other two cases follow in a similar vein. After reversing the signs of $\tau_{q+1}, \ldots, \tau_{m-1}$,

$$
T_{j} \rightsquigarrow\left\{\begin{array}{ll}
\tau_{1}-\tau_{j}-\tau_{j+1}+\tau_{m-1} & \text { if } 1 \leq j \leq q-1 \\
\tau_{1}-\tau_{q}+\tau_{m-1} & \text { if } j=q \\
\tau_{1}-\tau_{q+1}+\tau_{m-1} & \text { if } j=q+1 \\
\tau_{1}-\tau_{j-1}-\tau_{j}+\tau_{m-1} & \text { if } q+2 \leq j \leq m-1
\end{array} .\right.
$$

Next, we replace $\xi$ by $\xi-\left(\tau_{1}+\tau_{m-1}\right) / 2$. So we subtract from $T_{j}$ the term $\left(\tau_{1}+\tau_{m-1}\right) / 2$. This leads to

$$
T_{j} \rightsquigarrow \widetilde{T}_{j}:=\left\{\begin{array}{ll}
-\tau_{j}-\tau_{j+1} & \text { if } 1 \leq j \leq q-1 \\
-\tau_{q} & \text { if } j=q \\
-\tau_{q+1} & \text { if } j=q+1 \\
-\tau_{j-1}-\tau_{j} & \text { if } q+2 \leq j \leq m-1
\end{array} .\right.
$$

Let

$$
t_{j} \rightsquigarrow \widetilde{t}_{j}:=\left(\mathrm{IA}^{-1} \boldsymbol{\tau}\right)(j)=\left\{\begin{array}{ll}
\tau_{j}-\tau_{j+1} & \text { if } 1 \leq j \leq q-1 \\
\tau_{q} & \text { if } j=q \\
-\tau_{q+1} & \text { if } j=q+1 \\
\tau_{j-1}-\tau_{j} & \text { if } q+2 \leq j \leq m-1
\end{array} .\right.
$$


Now we replace $\xi$ by $-\xi$ so that

$$
\left(\omega+\mathrm{i}\left(2 \xi+T_{j}\right)\right)^{2}+t_{j}^{2} \rightsquigarrow\left(\omega-\mathrm{i}\left(2 \xi+\widetilde{T}_{j}\right)\right)^{2}+\widetilde{t}_{j}^{2} .
$$

For $j=q$ the last expression equals

$$
\left(\omega-\mathrm{i}\left(2 \xi+\tau_{q}\right)\right)^{2}+\tau_{q}^{2}=\omega^{2}-2 \mathrm{i} \omega\left(2 \xi+\tau_{q}\right)-(2 \xi)^{2}-4 \xi \tau_{q} .
$$

Next, we shift the integration with respect to $\tau_{q}$ by $\xi$. That is, we replace $\tau_{q}$ by $\tau_{q}-\xi$. Then we have

$$
\begin{aligned}
\omega^{2}-2 \mathrm{i} \omega\left(2 \xi+\tau_{q}\right)-(2 \xi)^{2}-4 \xi \tau_{q} & \rightsquigarrow \omega^{2}-2 \mathrm{i} \omega\left(\xi+\tau_{q}\right)-(2 \xi)^{2}-4 \xi\left(\tau_{q}-\xi\right) \\
& =\omega^{2}-2 \mathrm{i} \omega\left(\xi+\tau_{q}\right)-4 \xi \tau_{q} \\
& =(\omega-2 \mathrm{i} \xi)\left(\omega-2 \mathrm{i} \tau_{q}\right) .
\end{aligned}
$$

For $j=q+1$ we get in the end the expression $(\omega-2 \mathrm{i} \xi)\left(\omega-2 \mathrm{i} \tau_{q+1}\right)$.

For $1 \leq j \leq q-1$ we have

$$
\begin{aligned}
\left(\omega+\mathrm{i}\left(2 \xi-\tau_{j}-\tau_{j+1}\right)\right)^{2}+\left(\tau_{j}-\tau_{j+1}\right)^{2} & \rightsquigarrow\left(\omega-\mathrm{i}\left(2 \xi+\tau_{j}+\tau_{j+1}\right)\right)^{2}+\left(\tau_{j}-\tau_{j+1}\right)^{2} \\
& \rightsquigarrow\left(\omega-\mathrm{i}\left(\tau_{j}+\tau_{j+1}\right)\right)^{2}+\left(\tau_{j}-\tau_{j+1}\right)^{2} \\
& =\left(\omega-2 \mathrm{i} \tau_{j}\right)\left(\omega-2 \mathrm{i} \tau_{j+1}\right) .
\end{aligned}
$$

Similarly, for $q+2 \leq j \leq m-1$,

$$
\begin{aligned}
\left(\omega+\mathrm{i}\left(2 \xi-\tau_{j-1}-\tau_{j}\right)\right)^{2}+\left(\tau_{j-1}-\tau_{j}\right)^{2} & \rightsquigarrow\left(\omega-\mathrm{i}\left(2 \xi+\tau_{j-1}+\tau_{j}\right)\right)^{2}+\left(\tau_{j-1}-\tau_{j}\right)^{2} \\
& \rightsquigarrow\left(\omega-2 \mathrm{i} \tau_{j-1}\right)\left(\omega-2 \mathrm{i} \tau_{j}\right) .
\end{aligned}
$$

Finally, with $T_{m}=0$ and $t_{m}=\tau_{1}+\tau_{m-1}$,

$$
\begin{aligned}
\left(\omega+\mathrm{i}\left(2 \xi+T_{m}\right)\right)^{2}+\left(\tau_{1}+\tau_{m-1}\right)^{2} & \rightsquigarrow\left(\omega-\mathrm{i}\left(2 \xi-\tau_{1}-\tau_{m-1}\right)\right)^{2}+\left(\tau_{1}-\tau_{m-1}\right)^{2} \\
& \rightsquigarrow\left(\omega-2 \mathrm{i} \tau_{1}\right)\left(\omega-2 \mathrm{i} \tau_{m-1}\right) .
\end{aligned}
$$

B.3. Proof of the identity (3.28). We start out with the representation of the Laguerre polynomial as a contour integral in the complex plane $\mathbb{C}$, namely

$$
\mathcal{L}_{\ell}(x)=\frac{1}{2 \pi \mathrm{i}} \oint_{\Gamma} \frac{\mathrm{d} t}{(1-t) t^{\ell+1}} \exp [-x t /(1-t)] .
$$

Here, the contour $\Gamma$ is, say, a circle of radius $<1$, centered at the origin 0 , and with counter-clockwise orientation. Then, for any given pair $\xi, \tau \in \mathbb{R}$, we have

$$
\begin{aligned}
& \frac{1}{\sqrt{2 \pi}} \int_{\mathbb{R}} \mathrm{d} \omega \mathcal{L}_{\ell}((\omega-2 \mathrm{i} \xi)(\omega-2 \mathrm{i} \tau) / 2) \exp \left(-\omega^{2} / 4\right) \\
& \quad=\frac{1}{2 \pi \mathrm{i}} \oint_{\Gamma} \frac{\mathrm{d} t}{(1-t) t^{\ell+1}} \frac{1}{\sqrt{2 \pi}} \int_{\mathbb{R}} \mathrm{d} \omega \exp \left[-\omega^{2} / 4-(\omega-2 \mathrm{i} \xi)(\omega-2 \mathrm{i} \tau) t /(2(1-t))\right] .
\end{aligned}
$$

Now we observe

$$
-\frac{1}{4} \omega^{2}-\frac{(\omega-2 \mathrm{i} \xi)(\omega-2 \mathrm{i} \tau) t}{2(1-t)}=-\frac{1+t}{4(1-t)}\left(\omega-\frac{2 \mathrm{i}(\xi+\tau) t}{1+t}\right)^{2}+\frac{2 \xi \tau t}{1-t}-\frac{t^{2}(\xi+\tau)^{2}}{1-t^{2}}
$$


and perform the $\omega$-integration. This yields

$$
\left(\frac{4 \pi(1-t)}{1+t}\right)^{1 / 2} \exp \left[\frac{2 \xi \tau t}{1-t}-\frac{t^{2}(\xi+\tau)^{2}}{1-t^{2}}\right] .
$$

By the Cauchy integral formula (of the year 1831) we therefore get

$$
\begin{aligned}
\frac{1}{\sqrt{2 \pi}} & \int_{\mathbb{R}} \mathrm{d} \omega \mathcal{L}_{\ell}((\omega-2 \mathrm{i} \xi)(\omega-2 \mathrm{i} \tau) / 2) \exp \left(-\omega^{2} / 4\right) \\
& =\sqrt{2} \frac{1}{2 \pi \mathrm{i}} \oint_{\Gamma} \frac{\mathrm{d} t}{\sqrt{1-t^{2}} t^{\ell+1}} \exp \left[\frac{2 \xi \tau t}{1-t}-\frac{t^{2}(\xi+\tau)^{2}}{1-t^{2}}\right] \\
& =\left.\sqrt{2} \frac{1}{\ell !} \frac{\mathrm{d}^{\ell}}{\mathrm{d} t^{\ell}}\right|_{t=0} \frac{1}{\sqrt{1-t^{2}}} \exp \left[\frac{2 \xi \tau t}{1-t}-\frac{t^{2}(\xi+\tau)^{2}}{1-t^{2}}\right] .
\end{aligned}
$$

The Mehler formula (of the year 1866)

$$
\frac{1}{\sqrt{1-t^{2}}} \exp \left[\frac{2 \xi \tau t}{1-t}-\frac{t^{2}(\xi+\tau)^{2}}{1-t^{2}}\right]=\sum_{\ell=0}^{\infty} \frac{H_{\ell}(\xi) H_{\ell}(\tau)}{\ell !}\left(\frac{t}{2}\right)^{\ell}, \quad|t|<1
$$

now completes the proof of (3.28).

\section{References}

1. Birman, M.Sh., Solomyak, M.Z.: Estimates of singular numbers of integral operators, Russian Math. Surveys 32, 15-89 (1977) [Russian original: Uspekhi Mat. Nauk 32, $17-84$ (1977)]

2. Birman, M.Sh, Solomyak, M.Z.: Spectral Theory of Self-Adjoint Operators in Hilbert Space. Reidel, Dordrecht (1987)

3. Bratteli, O., Robinson, D.W.: Operator Algebras and Quantum Statistical Mechanics 2, 2nd edn. Springer, Berlin (1996)

4. Charles, L., Estienne, B.: Entanglement entropy and Berezin-Toeplitz operators. Commun. Math. Phys. 376, 521-554 (2020)

5. Eisert, J., Cramer, M., Plenio, M.B.: Area laws for the entanglement entropy: a review. Rev. Mod. Phys. 82, 277-306 (2010)

6. Elgart, A., Pastur, L., Shcherbina, M.: Large block properties of the entanglement entropy of disordered fermions. J. Stat. Phys. 166, 1092-1127 (2017)

7. Fock, V.: Bemerkung zur Quantelung des harmonischen Oszillators im Magnetfeld. Z. Phys. 47, 446-448 (1928). [in German]

8. Gioev, D., Klich, I.: Entanglement entropy of fermions in any dimension and the Widom conjecture. Phys. Rev. Lett. 96, 100503 (2006)

9. Helling, R., Leschke, H., Spitzer, W.: A special case of a conjecture by Widom with implications to fermionic entanglement entropy. Int. Math. Res. Notices 2011, 1451-1482 (2011)

10. Huang, K.: Statistical Mechanics, 2nd edn. Wiley, New York (1987)

11. Hupfer, T., Leschke, H., Warzel, S.: Upper bounds on the density of states of single Landau levels broadened by Gaussian random potentials. J. Math. Phys. 42, 5626-5641 (2001)

12. Kitaev, A., Preskill, J.: Topological entanglement entropy. Phys. Rev. Lett. 96, 110404 (2006)

13. Klich, I.: Lower entropy bounds and particle number fluctuations in a Fermi sea. J. Phys. A Math. Gen. 39, L85-L91 (2006)

14. Landau, L.: Diamagnetismus der Metalle. Z. Phys. 64, 629-637 (1930). [in German]

15. Leschke, H., Sobolev, A.V., Spitzer, W.: Large-scale behaviour of local and entanglement entropy of the free Fermi gas at any temperature. J. Phys. A: Math. Theor. 49 30LT04 (2016); Corrigendum: 50129501 (2017)

16. Leschke, H., Sobolev, A.V., Spitzer, W.: Scaling of Rényi entanglement entropies of the free Fermi-gas ground state: a rigorous proof. Phys. Rev. Lett. 112, 160403 (2014)

17. Leschke, H., Sobolev, A.V., Spitzer, W.: Trace formulas for Wiener-Hopf operators with applications to entropies of free fermionic equilibrium states. J. Funct. Anal. 273, 1049-1094 (2017) 
18. Levin, M., Wen, X.-G.: Detecting topological order in a ground state wave function. Phys. Rev. Lett. 96, 110405 (2006)

19. Müller, P., Schulte, R.: Stability of the enhanced area law of the entanglement entropy, preprint: arXiv:2004.02700

20. Müller, P., Pastur, L., Schulte, R.: How much delocalisation is needed for an enhanced area law of the entanglement entropy? Commun. Math. Phys 376, 649-679 (2020). https://doi.org/10.1007/s00220-02003784-3

21. Pastur, L., Shcherbina, M.: Szegö-type theorems for one-dimensional Schrödinger operator with random potential (smooth case). J. Math. Phys. Anal. Geom. [Zh. Mat. Fiz. Anal. Geom.] 14, 362-388 (2018)

22. Pastur, L., Slavin, V.: The absence of the selfaveraging property of the entanglement entropy of disordered free fermions in one dimension. J. Stat. Phys. 170, 207-220 (2017)

23. Peierls, R.: Surprises in Theoretical Physics. Princeton University Press, Princeton (1979)

24. Pfirsch, B., Sobolev, A.V.: Formulas of Szegő type for the periodic Schrödinger operator. Commun. Math. Phys. 358, 675-704 (2018)

25. Roccaforte, R.: On asymptotic expansions of traces for certain convolution operators. Trans. Am. Math. Soc. 285, 581-602 (1984)

26. Rodríguez, I.D., Sierra, G.: Entanglement entropy of integer quantum Hall states. Phys. Rev. B 80, 153303 (2009)

27. Rodríguez, I.D., Sierra, G.: Entanglement entropy of integer quantum Hall states in polygonal domains. J. Stat. Mech. 1012, P12033 (2010)

28. Simon, B.: Trace Ideals and Their Applications, 2nd edn. American Mathematical Society, Providence, Rhode Island (2005)

29. Sobolev, A.V.: Pseudo-Differential Operators with Discontinuous Symbols: Widom's Conjecture, Memoirs of the Amer. Math. Soc. 222(1043), Providence, Rhode Island, 2013

30. von Klitzing, K.: The quantized Hall effect. Rev. Mod. Phys. 58, 519-531 (1986)

31. Widom, H.: On a class of integral operators with discontinuous symbol. Oper. Theory Adv. Appl. 4, 477-500 (1982)

Communicated by J. Marklof 